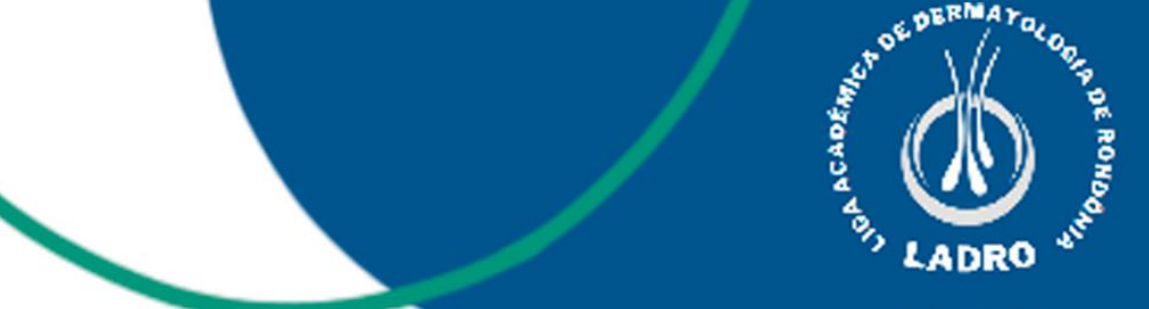

\title{
GUIA PRÁTICO DA HANSENIASE NA ATENÇÃo BÁSICA
}

$1^{\circ}$ Edição

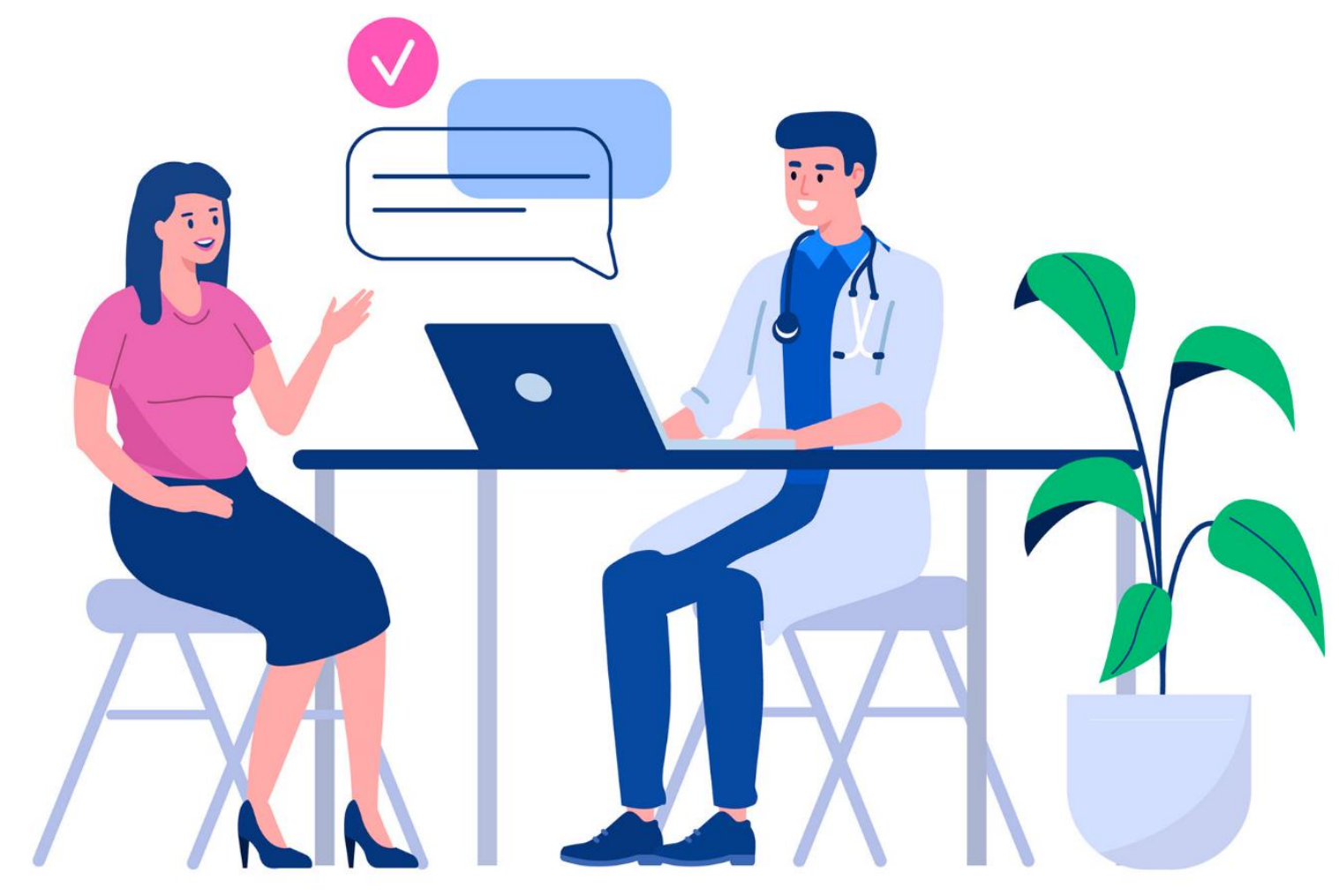

São José dos Pinhais

BRAZILIAN JOURNALS PUBLICAÇŌES DE PERIÓDICOS E EDITORA 2022 


\section{Cleber Queiroz Leite João Pedro Alencar Vieira Mariano \\ Brian França dos Santos Luís Marcelo Aranha Camargo}

\section{GUIA PRÁTICO DA HANSENÍASE NA ATENÇÃO BÁSICA}

$1^{\circ}$ Edição

Brazilian Journals Editora 2022 


\author{
2022 by Brazilian Journals Editora \\ Copyright (C) Brazilian Journals Editora \\ Copyright do Texto (C) 2022 Os Autores \\ Copyright da Edição (C) 2022 Brazilian Journals Editora \\ Editora Executiva: Barbara Luzia Sartor Bonfim \\ Diagramação: Sabrina B. \\ Imagens: Os Autores \\ Revisão: Os Autores
}

O conteúdo dos artigos e seus dados em sua forma, correção e confiabilidade são de responsabilidade exclusiva dos autores. Permitido o download da obra e o compartilhamento desde que sejam atribuídos créditos aos autores, mas sem a possibilidade de alterá-la de nenhuma forma ou utilizá-la para fins comerciais.

\title{
Conselho Editorial:
}

Prof ${ }^{a}$. Dra . Fátima Cibele Soares - Universidade Federal do Pampa, Brasil.

Prof. Dr. Gilson Silva Filho - Centro Universitário São Camilo, Brasil.

Prof. Msc. Júlio Nonato Silva Nascimento - Instituto Federal de Educação, Ciência e Tecnologia do Pará, Brasil.

Profä. Msc. Adriana Karin Goelzer Leining - Universidade Federal do Paraná, Brasil.

Prof. Msc. Ricardo Sérgio da Silva - Universidade Federal de Pernambuco, Brasil.

Prof. Esp. Haroldo Wilson da Silva - Universidade Estadual Paulista Júlio de Mesquita Filho, Brasil.

Prof. Dr. Orlando Silvestre Fragata - Universidade Fernando Pessoa, Portugal.

Prof. Dr. Orlando Ramos do Nascimento Júnior - Universidade Estadual de Alagoas, Brasil.

Profa . Drạ. Angela Maria Pires Caniato - Universidade Estadual de Maringá, Brasil.

Prof ${ }^{a}$. Drª . Genira Carneiro de Araujo - Universidade do Estado da Bahia, Brasil.

Prof. Dr. José Arilson de Souza - Universidade Federal de Rondônia, Brasil.

Profá. Msc. Maria Elena Nascimento de Lima - Universidade do Estado do Pará, Brasil.

Prof. Caio Henrique Ungarato Fiorese - Universidade Federal do Espírito Santo, Brasil.

Prof ${ }^{a}$. Dra . Silvana Saionara Gollo - Instituto Federal de Educação, Ciência e Tecnologia do

Rio Grande do Sul, Brasil.

Prof $^{a}$. Dr a . Mariza Ferreira da Silva - Universidade Federal do Paraná, Brasil.

Prof. Msc. Daniel Molina Botache - Universidad del Tolima, Colômbia.

Prof. Dr. Armando Carlos de Pina Filho- Universidade Federal do Rio de Janeiro, Brasil.

Prof. Dr. Hudson do Vale de Oliveira- Instituto Federal de Educação, Ciência e Tecnologia de Roraima, Brasil.

Profa ${ }^{a}$. Msc. Juliana Barbosa de Faria - Universidade Federal do Triângulo Mineiro, Brasil.

Profa ${ }^{-}$. Esp. Marília Emanuela Ferreira de Jesus - Universidade Federal da Bahia, Brasil.

Prof. Msc. Jadson Justi - Universidade Federal do Amazonas, Brasil.

Prof ${ }^{a}$. Dra . Alexandra Ferronato Beatrici - Instituto Federal de Educação, Ciência e

Tecnologia do Rio Grande do Sul, Brasil.

Prof ${ }^{a}$. Msc. Caroline Gomes Mâcedo - Universidade Federal do Pará, Brasil.

Prof. Dr. Dilson Henrique Ramos Evangelista - Universidade Federal do Sul e Sudeste do Pará, Brasil.

Prof. Dr. Edmilson Cesar Bortoletto - Universidade Estadual de Maringá, Brasil.

Prof. Msc. Raphael Magalhães Hoed - Instituto Federal do Norte de Minas Gerais, Brasil.

Profa. Msc. Eulália Cristina Costa de Carvalho - Universidade Federal do Maranhão, Brasil.

Prof. Msc. Fabiano Roberto Santos de Lima - Centro Universitário Geraldo di Biase, Brasil.

Prof ${ }^{a}$. Dr ${ }^{a}$. Gabrielle de Souza Rocha - Universidade Federal Fluminense, Brasil. 
Prof. Dr. Helder Antônio da Silva, Instituto Federal de Educação do Sudeste de Minas Gerais, Brasil.

Prof ${ }^{a}$. Esp. Lida Graciela Valenzuela de Brull - Universidad Nacional de Pilar, Paraguai.

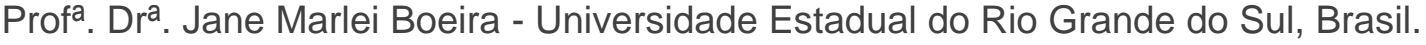

Prof ${ }^{-}$. Dr ${ }^{\mathrm{a}}$. Carolina de Castro Nadaf Leal - Universidade Estácio de Sá, Brasil.

Prof. Dr. Carlos Alberto Mendes Morais - Universidade do Vale do Rio do Sino, Brasil.

Prof. Dr. Richard Silva Martins - Instituto Federal de Educação, Ciência e Tecnologia Sul Rio Grandense, Brasil.

Prof ${ }^{a}$. Drª . Ana Lídia Tonani Tolfo - Centro Universitário de Rio Preto, Brasil.

Prof. Dr. André Luís Ribeiro Lacerda - Universidade Federal de Mato Grosso, Brasil.

Prof. Dr. Wagner Corsino Enedino - Universidade Federal de Mato Grosso, Brasil.

Prof ${ }^{a}$. Msc. Scheila Daiana Severo Hollveg - Universidade Franciscana, Brasil.

Prof. Dr. José Alberto Yemal - Universidade Paulista, Brasil.

Profa . Dra . Adriana Estela Sanjuan Montebello - Universidade Federal de São Carlos, Brasil.

Profa . Msc. Onofre Vargas Júnior - Instituto Federal de Educação, Ciência e Tecnologia

Goiano, Brasil.

Prof ${ }^{\text {. Dr }}$. Rita de Cássia da Silva Oliveira - Universidade Estadual de Ponta Grossa, Brasil.

Prof ${ }^{a}$. Dra . Leticia Dias Lima Jedlicka - Universidade Federal do Sul e Sudeste do Pará,

Brasil.

Profa . Dra . Joseina Moutinho Tavares - Instituto Federal da Bahia, Brasil

Prof. Dr. Paulo Henrique de Miranda Montenegro - Universidade Federal da Paraíba, Brasil.

Prof. Dr. Claudinei de Souza Guimarães - Universidade Federal do Rio de Janeiro, Brasil.

Prof ${ }^{a}$. Dr ${ }^{a}$. Christiane Saraiva Ogrodowski - Universidade Federal do Rio Grande, Brasil.

Prof ${ }^{a}$. Dr $\stackrel{a}{\text { a }}$. Celeide Pereira - Universidade Tecnológica Federal do Paraná, Brasil.

Prof ${ }^{a}$. Msc. Alexandra da Rocha Gomes - Centro Universitário Unifacvest, Brasil.

Prof ${ }^{a}$. Dr ${ }^{a}$. Djanavia Azevêdo da Luz - Universidade Federal do Maranhão, Brasil.

Prof. Dr. Eduardo Dória Silva - Universidade Federal de Pernambuco, Brasil.

Prof ${ }^{a}$. Msc. Juliane de Almeida Lira - Faculdade de Itaituba, Brasil.

Prof. Dr. Luiz Antonio Souza de Araujo - Universidade Federal Fluminense, Brasil.

Prof. Dr. Rafael de Almeida Schiavon - Universidade Estadual de Maringá, Brasil.

Prof ${ }^{a}$. Dr ${ }^{a}$. Rejane Marie Barbosa Davim - Universidade Federal do Rio Grande do Norte, Brasil.

Prof. Msc. Salvador Viana Gomes Junior - Universidade Potiguar, Brasil.

Prof. Dr. Caio Marcio Barros de Oliveira - Universidade Federal do Maranhão, Brasil.

Prof. Dr. Cleiseano Emanuel da Silva Paniagua - Instituto Federal de Educação, Ciência e

Tecnologia de Goiás, Brasil.

Prof ${ }^{a}$. Dr ${ }^{\mathrm{a}}$. Ercilia de Stefano - Universidade Federal Fluminense, Brasil. 


\begin{tabular}{|l|}
\hline Dados Internacionais de Catalogação na Publicação (CIP) \\
\hline L533g Leite, Cleber Queiroz \\
Guia prático da Hanseníase na atenção básica / Cleber Queiroz \\
Leite, João Pedro Alencar Vieira Mariano, Brian França dos \\
Santos, Luís Marcelo Aranha Camargo. São José dos Pinhais: \\
Editora Brazilian Journals, 2022. \\
35 p. \\
Formato: PDF \\
Requisitos de sistema: Adobe Acrobat Reader \\
Modo de acesso: World Wide Web \\
Inclui: Bibliografia \\
ISBN: 978-65-81028-17-6 \\
DOI: 10.35587/brj.ed.0001350 \\
1. Saúde. 2. Hansenĺase. I. Leite, Cleber Queiroz. II. Mariano, \\
João Pedro Alencar Vieira. III. Dos Santos, Brian França. IV. \\
Camargo, Luís Marcelo Aranha. V. Título. \\
\hline
\end{tabular}

Brazilian Journals Editora

São José dos Pinhais - Paraná - Brasil

www.brazilianjournals.com.br

editora@brazilianjournals.com.br 


\section{AUTORES}

João Pedro Alencar Vieira Mariano - Centro Universitário São Lucas. E-mail: joao.peddrro2@gmail.com Orcid: 0000-0002-8285-3984

Cleber Queiroz Leite - Centro Universitário São Lucas. E-mail: cleberqueiroz05@hotmail.com - Orcid: 00000002-7847-1166

Brian França dos Santos - Universidade Iguaçu. E-mail:drbrianfranca@hotmail.com - Orcid: 0000-0003$1574-630 X$

Bruna de Brito Fogaça - Centro Universitário São Lucas. E-mail: bruninhabrito98@ gmail.com - Orcid: 00000002-6565-2013

Bruna Milani - Centro Universitário Aparício Carvalho - FIMCA. E-mail: bruna_milani2000@ hotmail.com Orcid: 0000-0002-0566-5226

Willyam Patricky Fernandes da Costa - Centro Universitário São Lucas E-mail: willyampatricky@gmail.com - Orcid: 0000-0003-4908-7410

Aline Cardoso Silva - Faculdade Metropolitana (UNNESA). E-mail: alinecsilva755@gmail.com - Orcid: 00000001-8255-1583

Lineker Souza do Amaral - Centro Universitário São Lucas. E-mail: linnekerferracine@gmail.com- Orcid: 0000-0003-2656-8772

Marcela Fernandes Lúcio - Faculdade Metropolitana (UNNESA). E-mail: marcelalucio3@ hotmail.com Orcid: 0000-0002-3670-3475

Nando Rabelo Mesquita - Centro Universitário São Lucas. E-mail: nando.rabelo22@ gmail.com - Orcid: 00000002-2002-6369

Izabella Gurgel do Amaral Pini - Centro Universitário São Lucas. E-mail: izabellapini@gmail.com - Orcid: 0000-0003-0778-7574

Thayslane de Carvalho Barbosa - Centro Universitário São Lucas. E-mail: thays-decarvalho@ hotmail.com Orcid: 0000-0003-4321-9291

Renata Mesquita Kestering - Centro Universitário São Lucas. E-mail: renatamesquit@hotmail.com - Orcid: 0000-0001-6215-9330

Cassia Nascimento Tavares - Centro Universitário São Lucas. E-mail: cassia_17_1@hotmail.com - Orcid: 0000-0003-4761-4993

Giovana Maria Facchin Silva Chaves - Centro Universitário Aparício Carvalho - FIMCA. Email:giovanachavesf@gmail.com - Orcid: 0000-0002-6902-5404

Guilherme Tadeu Souza Batista - Centro Universitário São Lucas. E-mail: guilherme.tb@ oulook.com - Orcid: 0000-0002-8050-7391

Renato Claros Lima - Centro Universitário Aparício Carvalho - FIMCA. E-mail: renato.claros@ hotmail.com Orcid: 0000-0002-8800-0712

João Victor Rulnix Sena - Centro Universitário Aparício Carvalho - FIMCA. E-mail: joaovictor.sena99@gmail.com - Orcid: 0000-0003-4674-3486

Mariana Gabrielle Silva de Lima - Centro Universitário Aparício Carvalho - FIMCA. E-mail: marianagslima3@gmail.com - Orcid: 0000-0002-5761-5693

Luís Marcelo Aranha Camargo - Centro Universitário São Lucas. E-mail: spider@icbusp.org - Orcid: 00000001-9486-6195 


\section{SUMÁRIO}

\begin{tabular}{|c|c|c|c|}
\hline 01 & 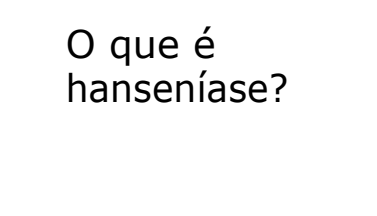 & 02 & 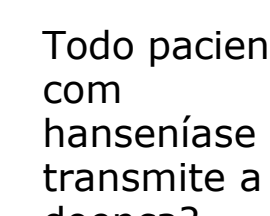 \\
\hline 03 & 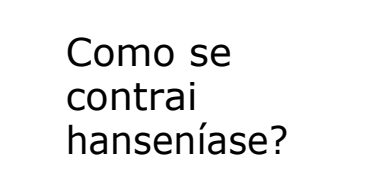 & 04 & 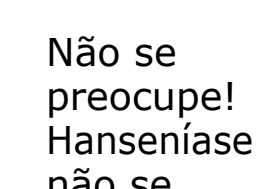 \\
\hline 5 & 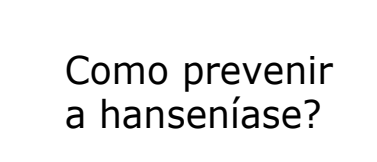 & 06 & 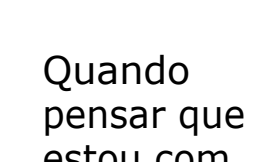 \\
\hline 07 & 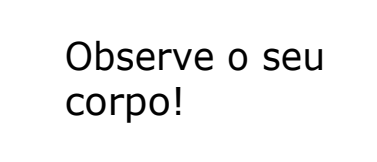 & 09 & 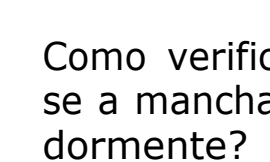 \\
\hline 10 & 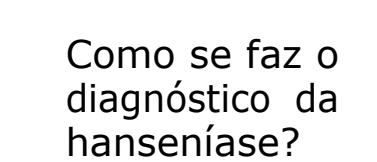 & 11 & 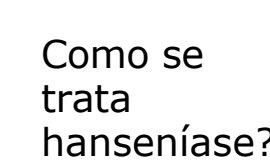 \\
\hline 12 & 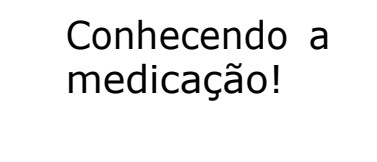 & 15 & \\
\hline 17 & 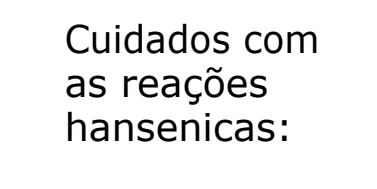 & 18 & oprever \\
\hline 21 & & 22 & \\
\hline 24 & 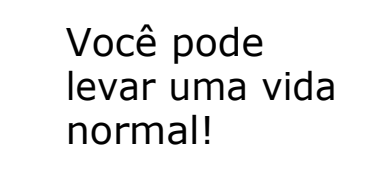 & 25 & \\
\hline
\end{tabular}




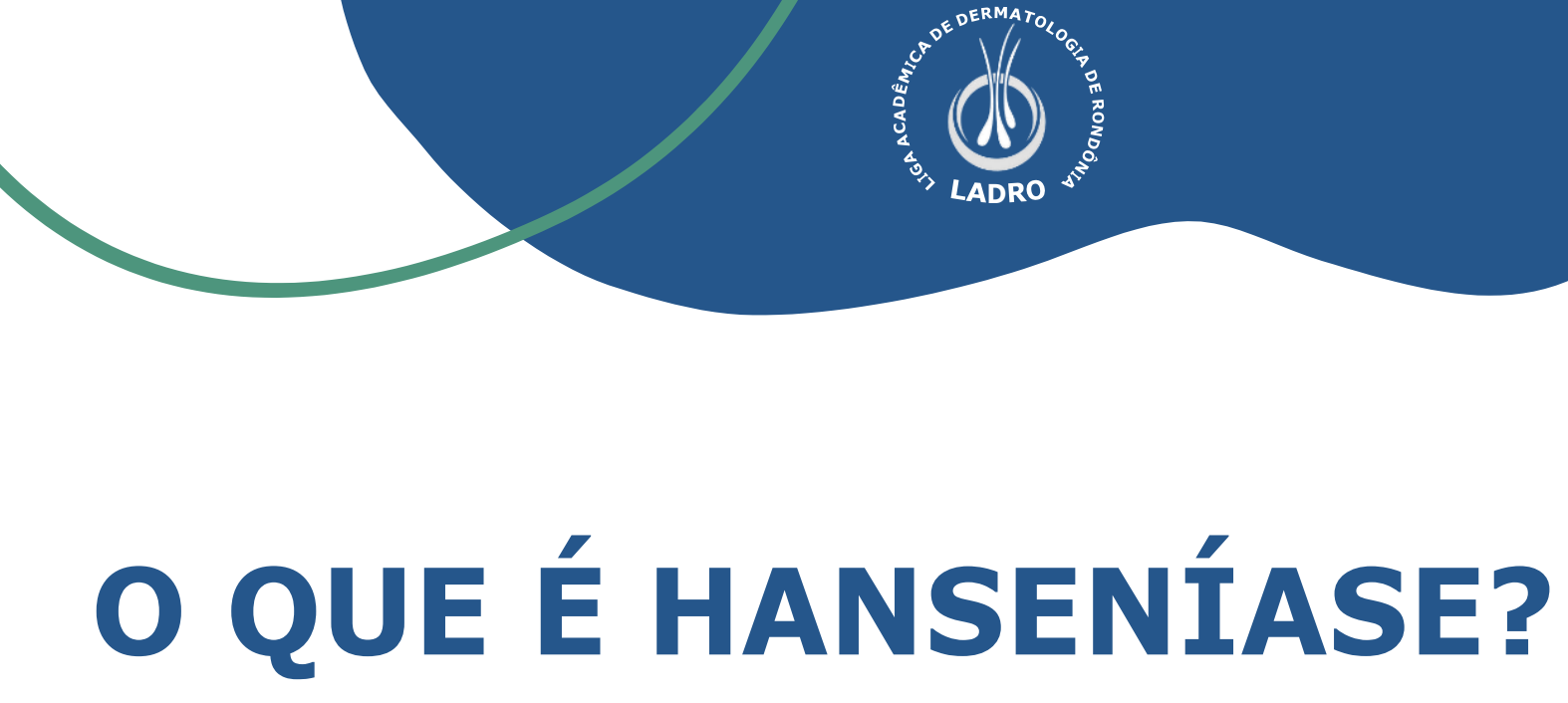

\section{O QUE É HANSENÍASE?}

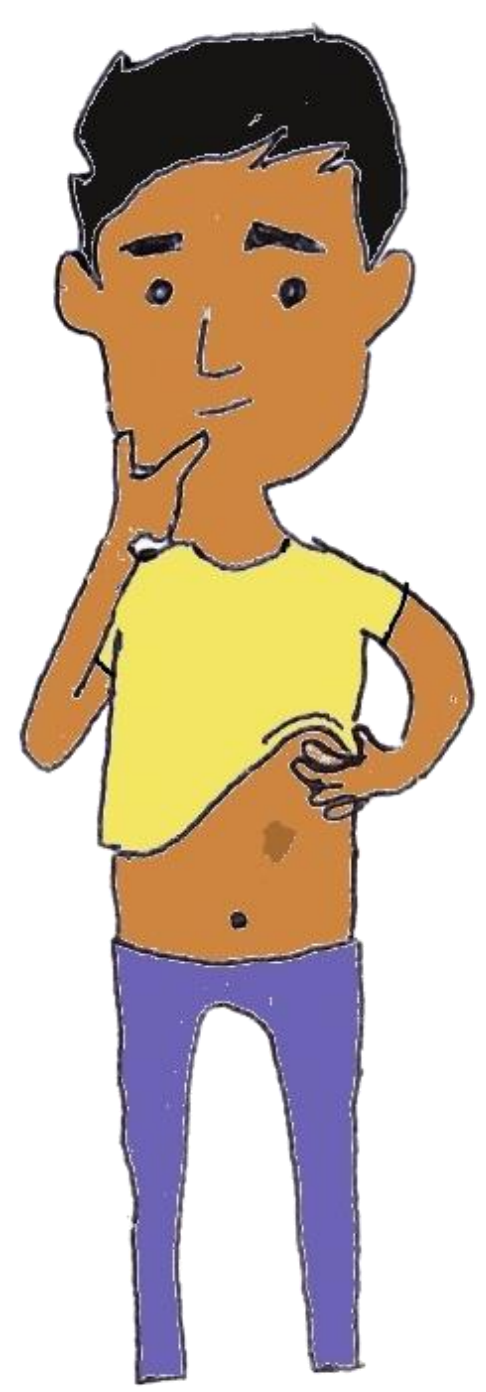

Antigamente conhecida como lepra, a hanseníase é uma doença infecciosa e altamente contagiosa, causada pela bactéria Mycobacterium leprae, que atinge principalmente a pele, nervos periféricos e mucosas.

Caracterizada pela evolução crônica, em caso de diagnóstico tardio pode gerar lesões neurais irreversíveis e, como consequências, deformidades e incapacidade física. Atinge ambos os sexos, sendo menos comum em crianças.

O tratamento é oferecido gratuitamente nas Unidades de Saúde e leva a cura da doença. 


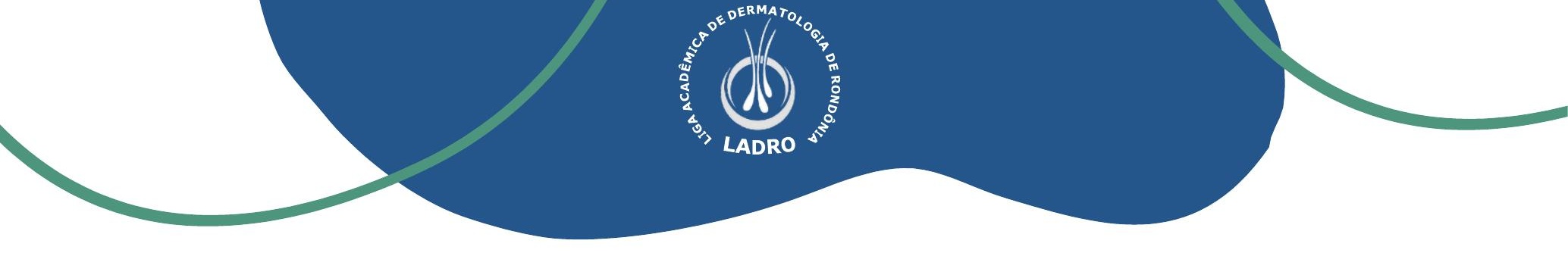

\section{TODO PACIENTE COM HANSENÍASE TRANSMITE A DOENÇA?}

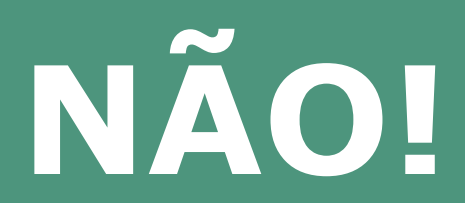

Somente os pacientes multibacilares, ou seja, os que possuem muitas bactérias e lesões, e que NÃO estejam em tratamento, transmitem a doença. 


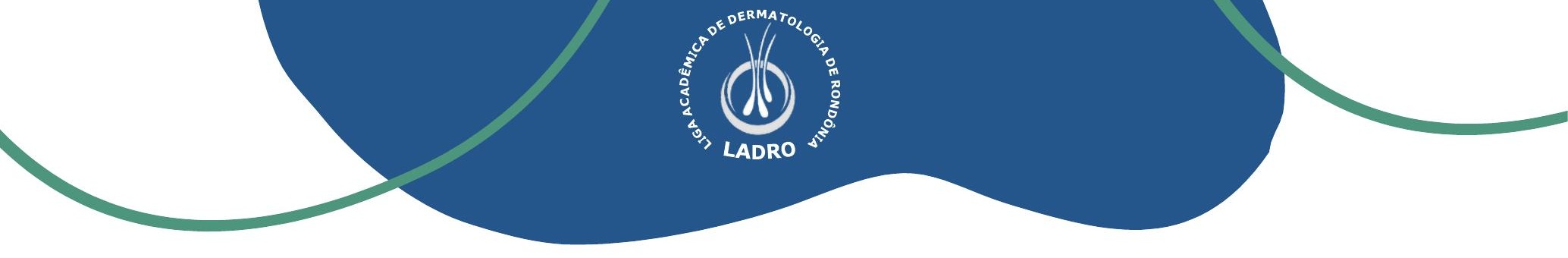

\section{COMO SE CONTRAI HANSENÍASE?}

A transmissão ocorre de pessoa para pessoa, embora a maioria dos indivíduos possua imunidade natural à doença, ou seja, podem se infectar, mas não adoecem.

Precárias condições e desnutrição são fatores que favorecem o adoecimento por hanseníase. Além disso, o contato ou o consumo de carne de tatu pode levar o ser humano a contrair hanseníase.

Pacientes multibacilares, no estágio avançado da doença, na forma clínica virchowiana e dimorfa, quando não tratados, transmitem o bacilo por meio das gotículas de saliva, quando falam, espirram ou tossem. Assim, as pessoas que convivem com estes pacientes respiram o bacilo. $\mathrm{O}$ bacilo aspirado, pode

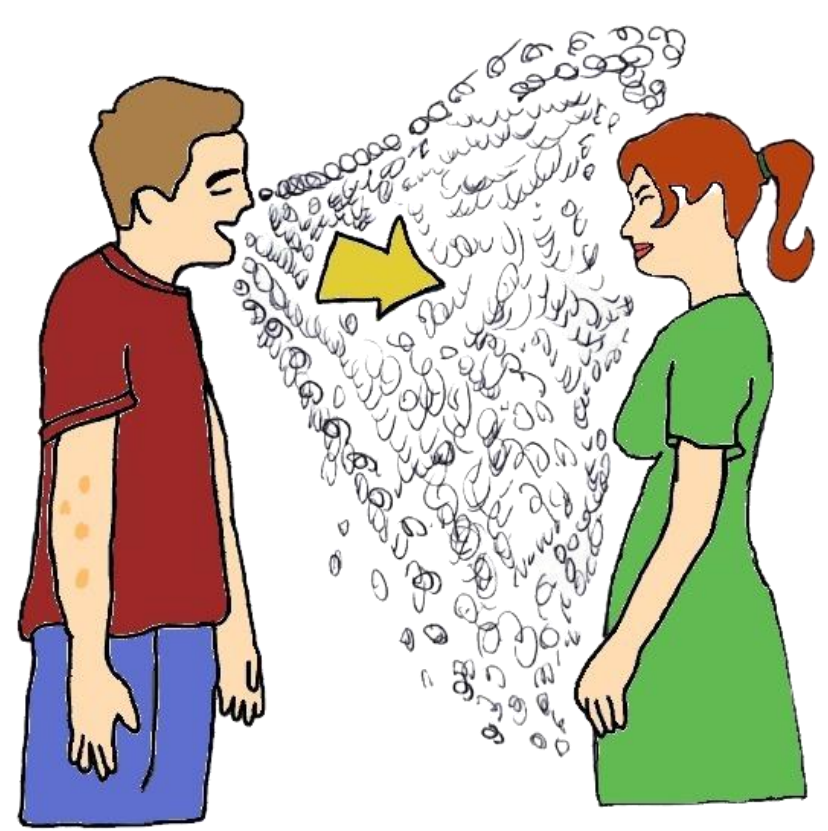
se instalar no organismo e vir a provocar a doença posteriormente. Ambientes fechados, com pouca ventilação e ausência de luz solar, favorecem a infecção e o adoecimento.

Vale ressaltar que a hanseníase não é hereditária, nem congênita. 



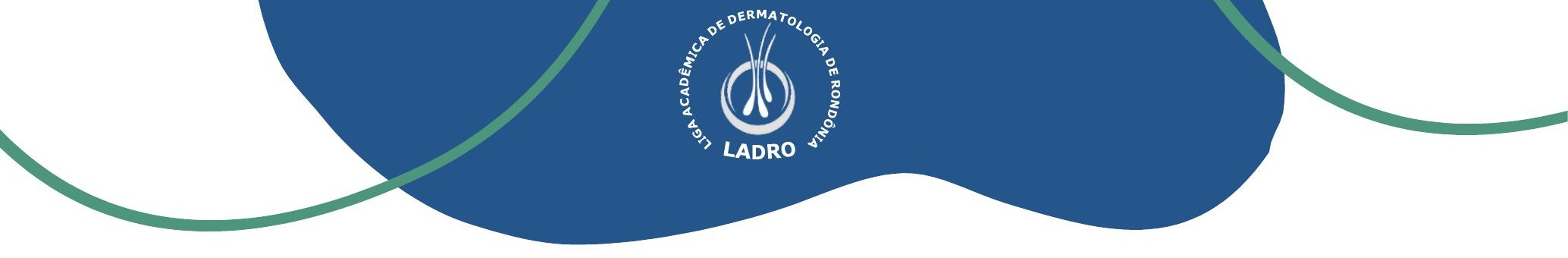

\section{COMO PREVENIR A HANSENÍASE?}

A melhor forma de prevenção é o diagnóstico precoce e o tratamento adequado. Hábitos saudáveis, como a prática de atividade física e a alimentação adequada, evitando álcool, associados a condições de higiene, contribuem para dificultar o adoecimento pela hanseníase.

A vacina BCG pode melhorar a defesa dos contatos do paciente, interrompendo a cadeia de transmissão da doença.

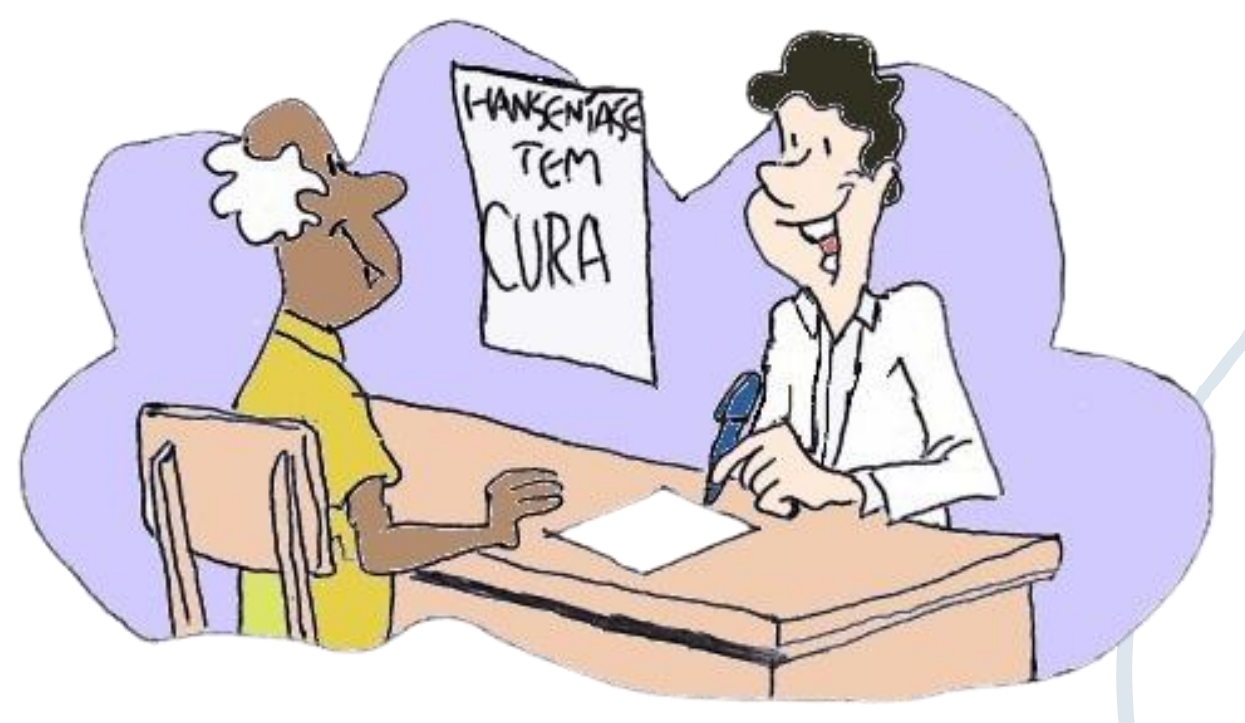




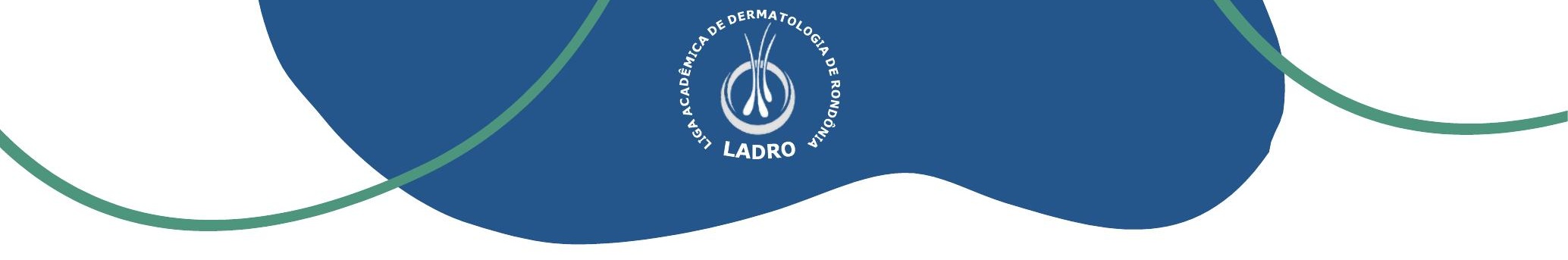

\section{QUANDO PENSAR QUE ESTOU COM HANSENÍASE?}

Os principais sintomas são a presença de áreas e/ou manchas na pele, que podem ser esbranquiçadas, acobreadas, amarronzadas ou avermelhadas, nas quais há alteração na sensibilidade ao frio, ao calor, a dor e ao toque. Ainda, pode haver áreas da pele com queda de pelos, diminuição de suor, presença de nódulos, e sensação de formigamento, principalmente nas mãos e pés.

Caso perceba-se alguma dessas alterações, deve-se considerar o diagnóstico de hanseníase e procurar atendimento na Unidade de Saúde para confirmação.
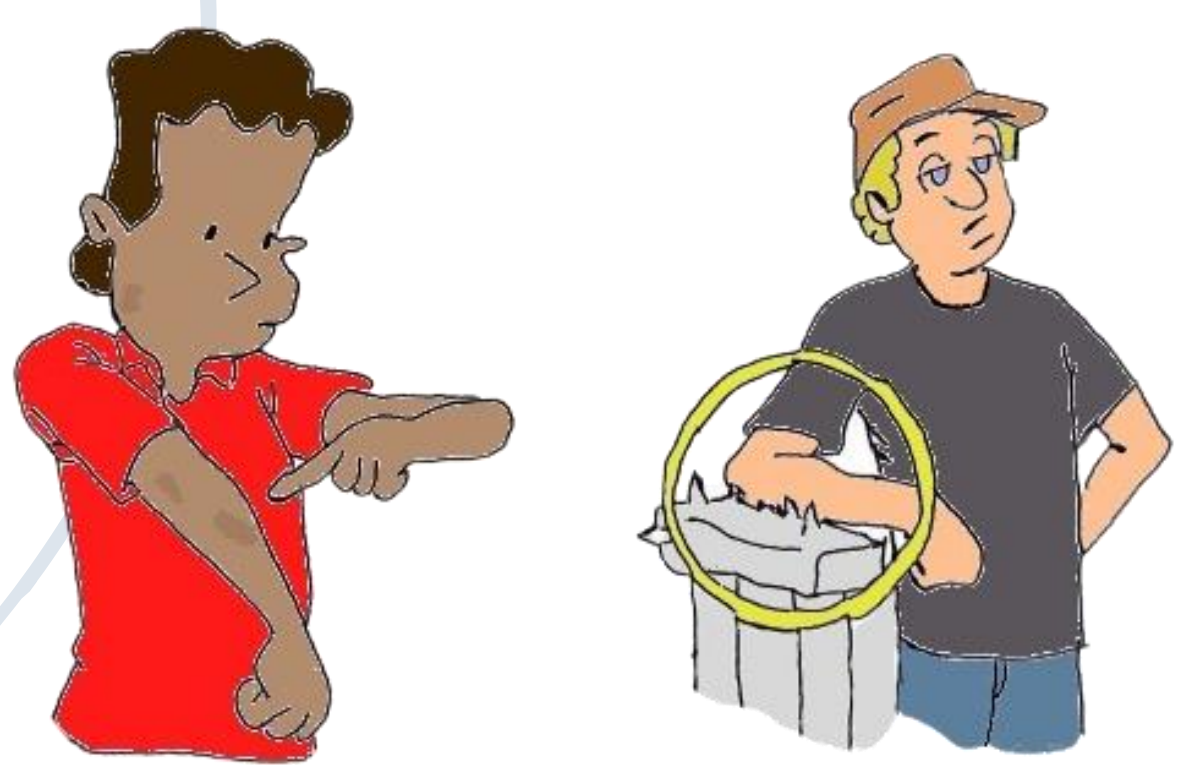


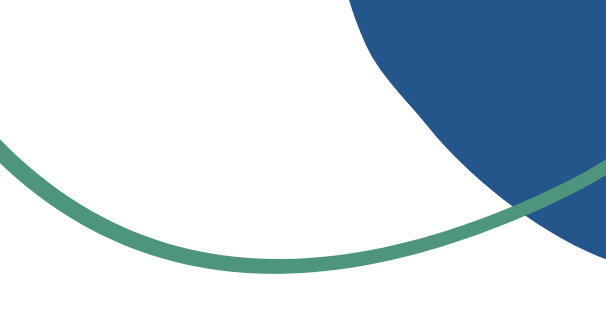

\section{OBSERVE O SEU CORPO!}

Os principais sinais e sintomas da hanseníase são:

- Áreas da pele, ou manchas esbranquiçadas (hipocrômicas), acastanhadas ou avermelhadas, com alterações de sensibilidade ao calor, e/ou ao tato, e/ou dolorosa;

- Formigamentos, choques e câimbras nos braços e pernas, que evoluem para dormência - a pessoa se queima ou se machuca sem perceber;

- Pápulas, tubérculos e nódulos (caroços), normalmente sem sintomas;

- Diminuição ou queda de pelos, localizada ou difusa, especialmente nas sobrancelhas (madarose);

- Pele infiltrada (avermelhada), com diminuição ou ausência de suor no local.

Além dos sinais e sintomas mencionados, pode-se observar:

- Dor, choque ou espessamento de nervos das mãos e pernas;

- Diminuição ou perda de sensibilidade nas áreas dos nervos afetados, principalmente nos olhos, mãos e pés;
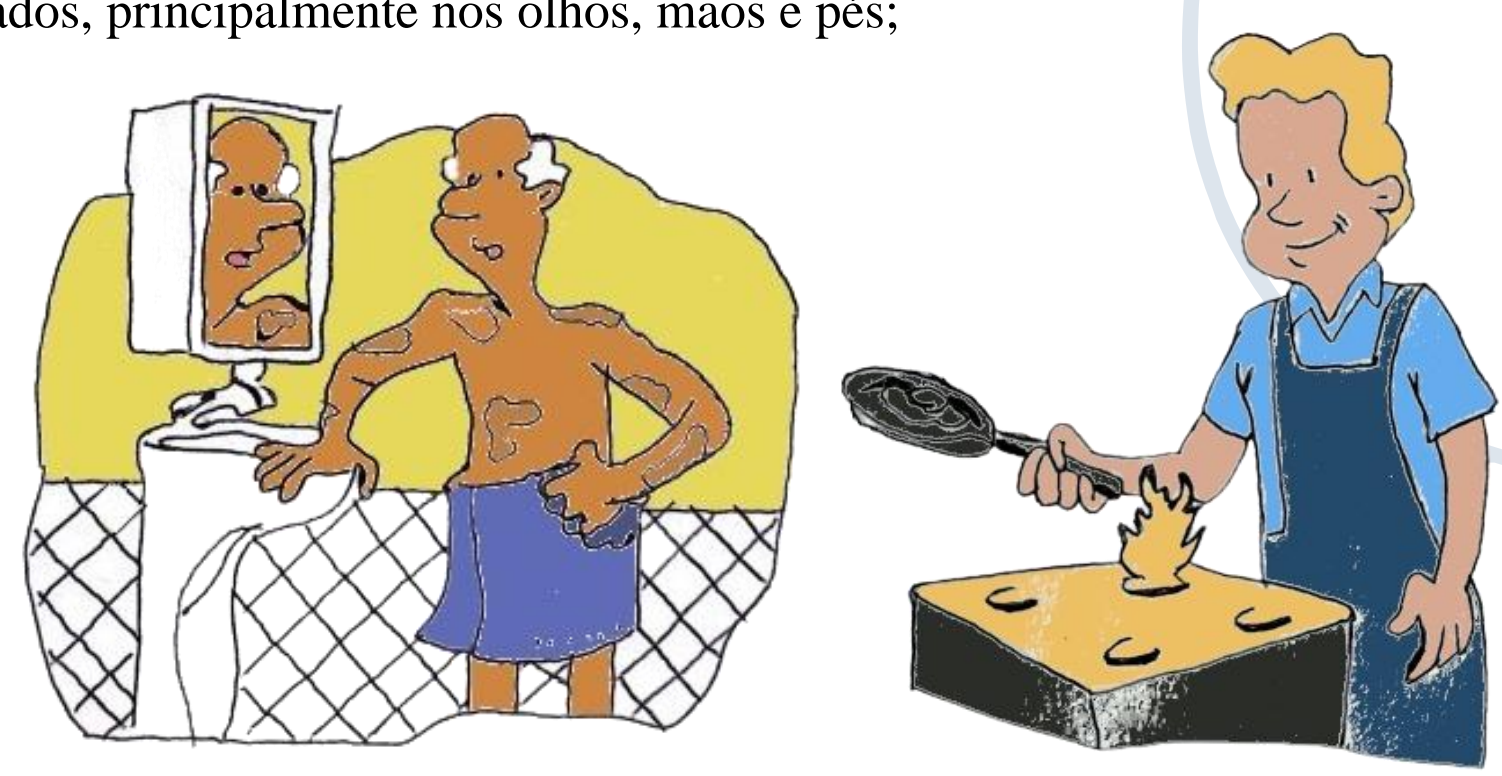


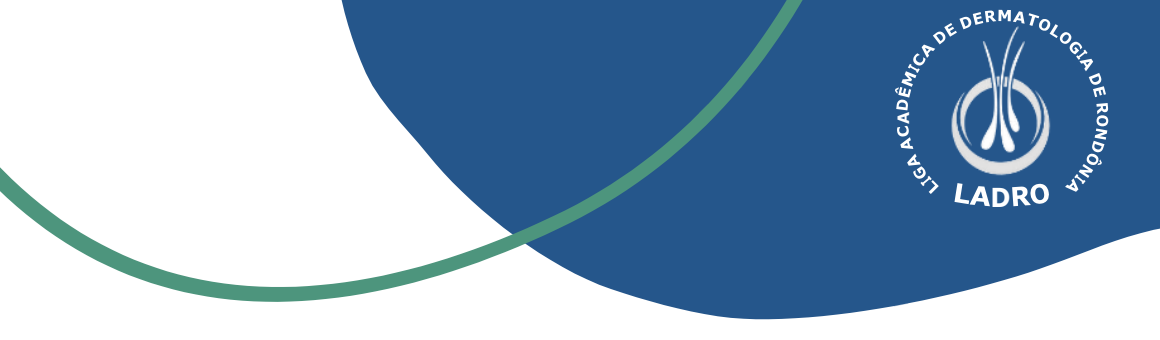

\section{OBSERVE O SEU CORPO!}

- Diminuição ou perda de força nos músculos, principalmente nos membros superiores e inferiores e, por vezes, nas pálpebras;

- Inchaço de mãos e pés com cianose (arroxeamento dos dedos) e ressecamento da pele;

- Febre e artralgia, associados a caroços dolorosos, desaparecimento repentino;

- Aparecimento repentino de manchas dormentes com dor nos nervos dos cotovelos (ulnares), joelhos (fibulares comuns) e tornozelos (tibiais posteriores ural);

- Entupimento, malcheiro, feridas e ressecamento do nariz;

- Ressecamento e sensação de areia nos olhos.
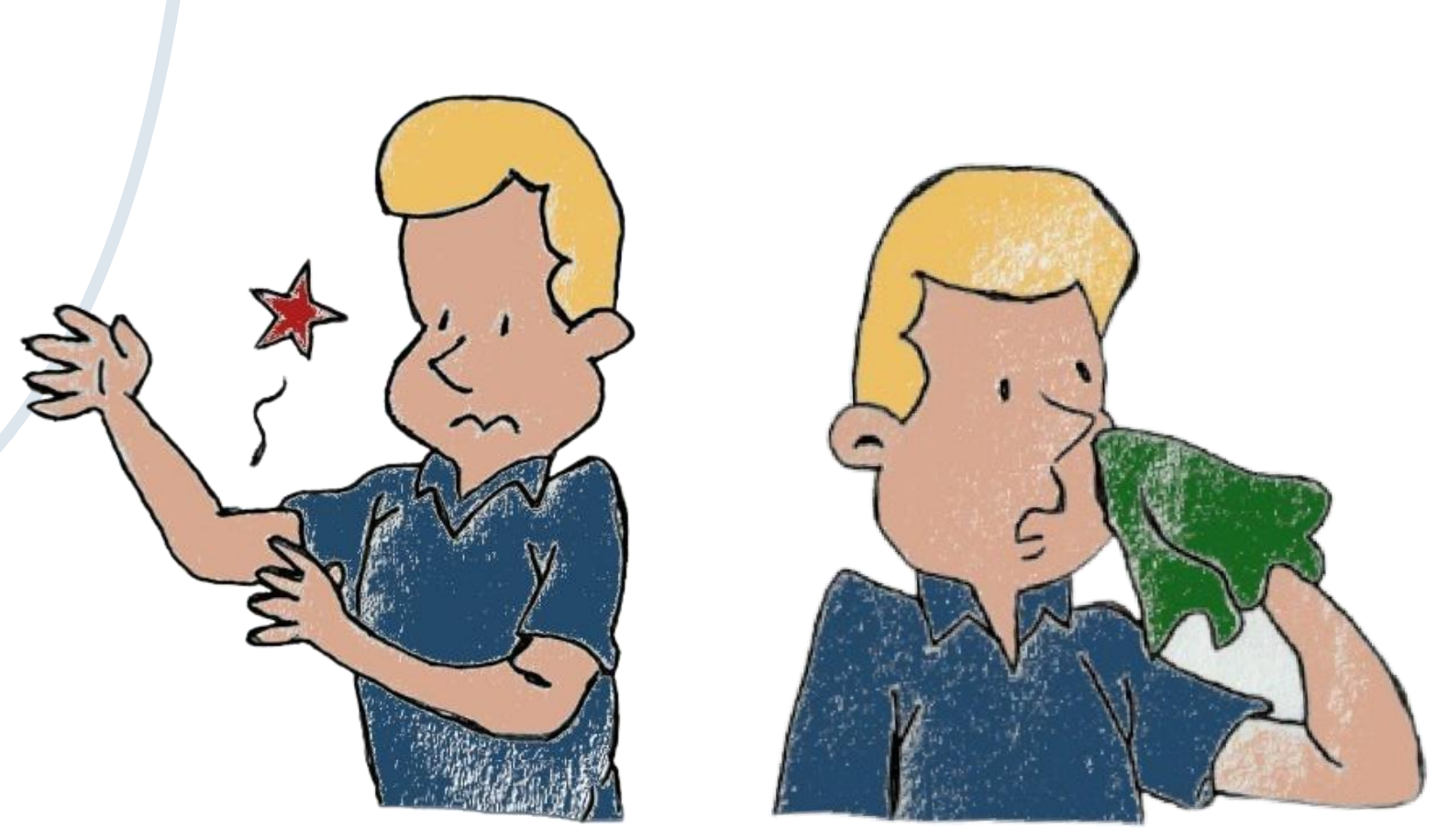


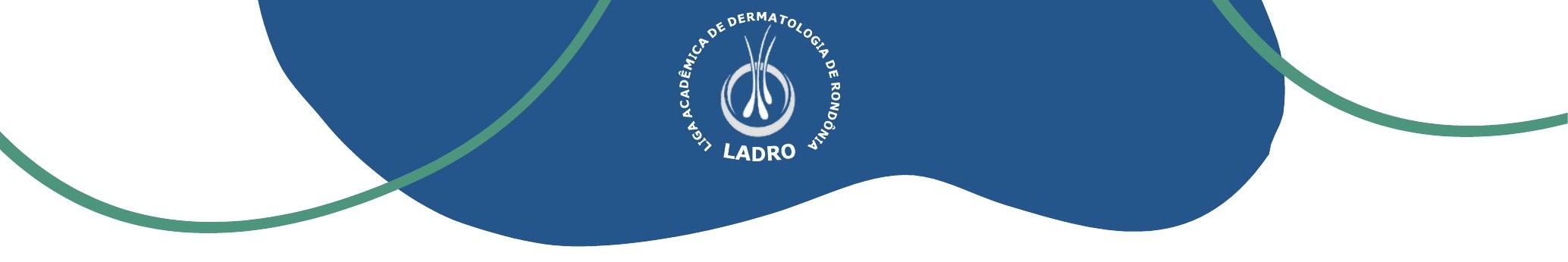

\section{COMO VERIFICAR SE}

A ÁREA OU MANCHA
É DORMENTE?

As áreas não apresentam lesões. Quanto muito a perda de pelos e ressecamento da pele por falta de suor. As manchas são mais claras ou mais avermelhadas que a pele.

Podem aparecer caroços. Essas áreas e/ou manchas ficam "dormentes", ou seja, geralmente não sentem a diferença entre o quente e o frio, às vezes, não sentem dor.
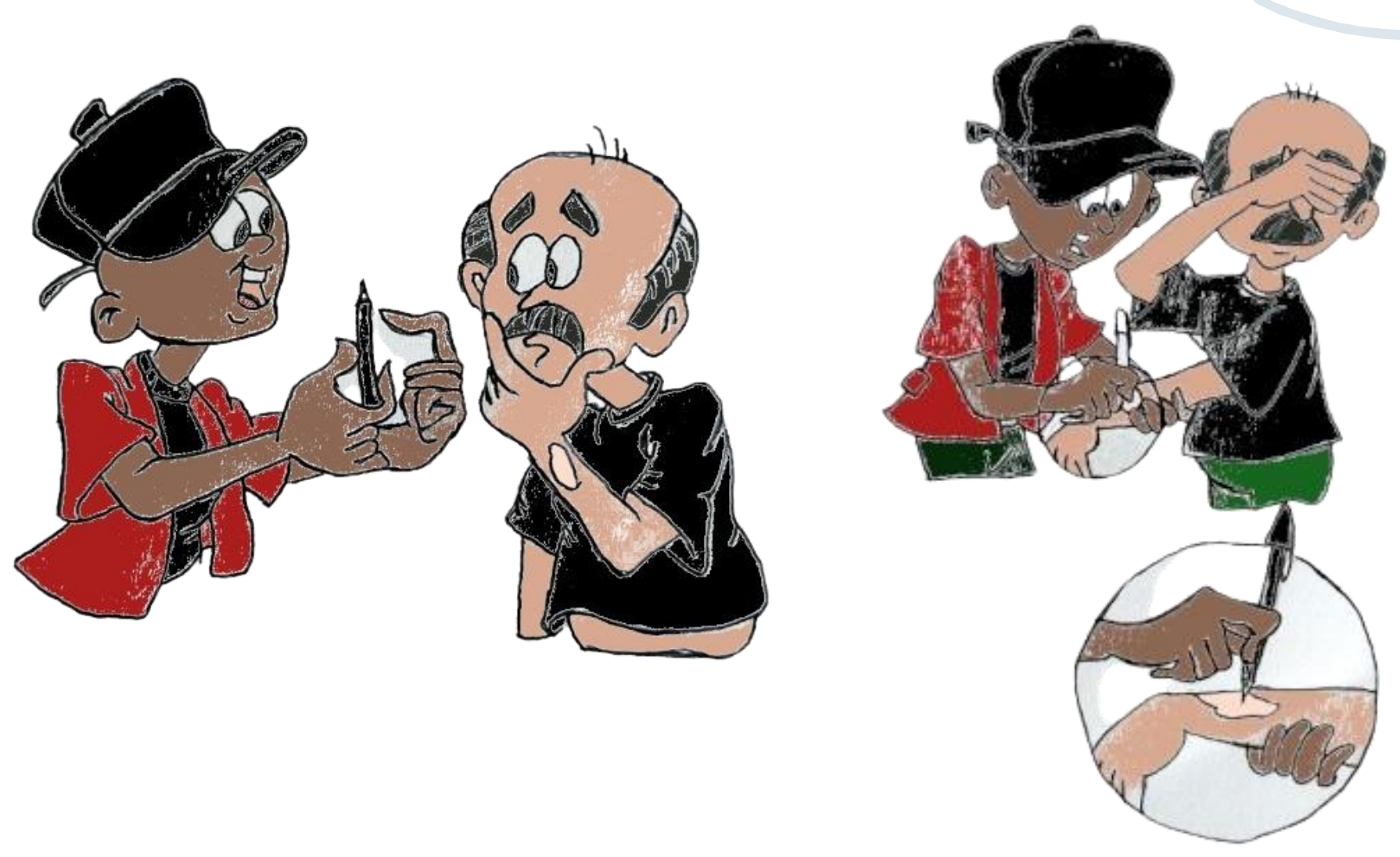

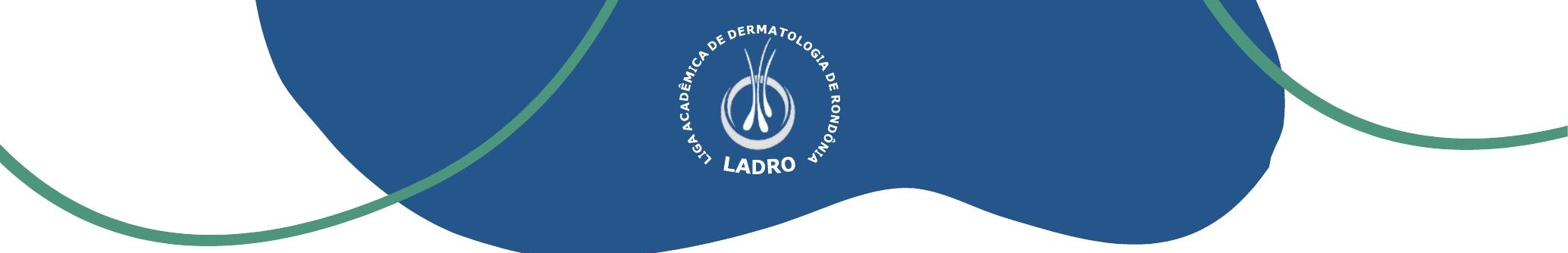

\section{COMO SE FAZ O DIAGNÓSTICO DA HANSENÍASE?}

O diagnóstico é especialmente clínico e epidemiológico, tem a finalidade de identificar lesões na pele e possíveis áreas de sensibilidades.

O raspado dérmico de pacientes com suspeita clínica de hanseníase é um procedimento laboratorial rápido, de baixo custo, menos invasivo e que não necessita de tecnologia avançada. No entanto não é muito sensivel para o diagnóstico da doença.

Pode, também, ser feito biópsia das lesões (retirada de um pequeno pedaço da pele sobre anestesia local) para exame ao microscópio.

De acordo com o $8^{\circ}$ Comitê de Hanseníase da Organização Mundial de Saúde (OMS), em 2012, o diagnóstico de hanseníase deve ser suspeitado em indivíduos que apresentem os seguintes sinais ou sintomas:

- Áreas ou manchas pálidas (hipocrômicas) ou avermelhadas na pele com perda ou redução da sensibilidade;

- Sensação de "adormecimento" ou "formigamento" em mãos ou pés;

- Fraqueza em mãos, pés ou pálpebras;

- Dor ou sensibilidade na topografia de nervos;

- Aumento do volume da face ou lóbulos da orelha;

- Feridas indolores ou queimaduras em pés ou mãos.

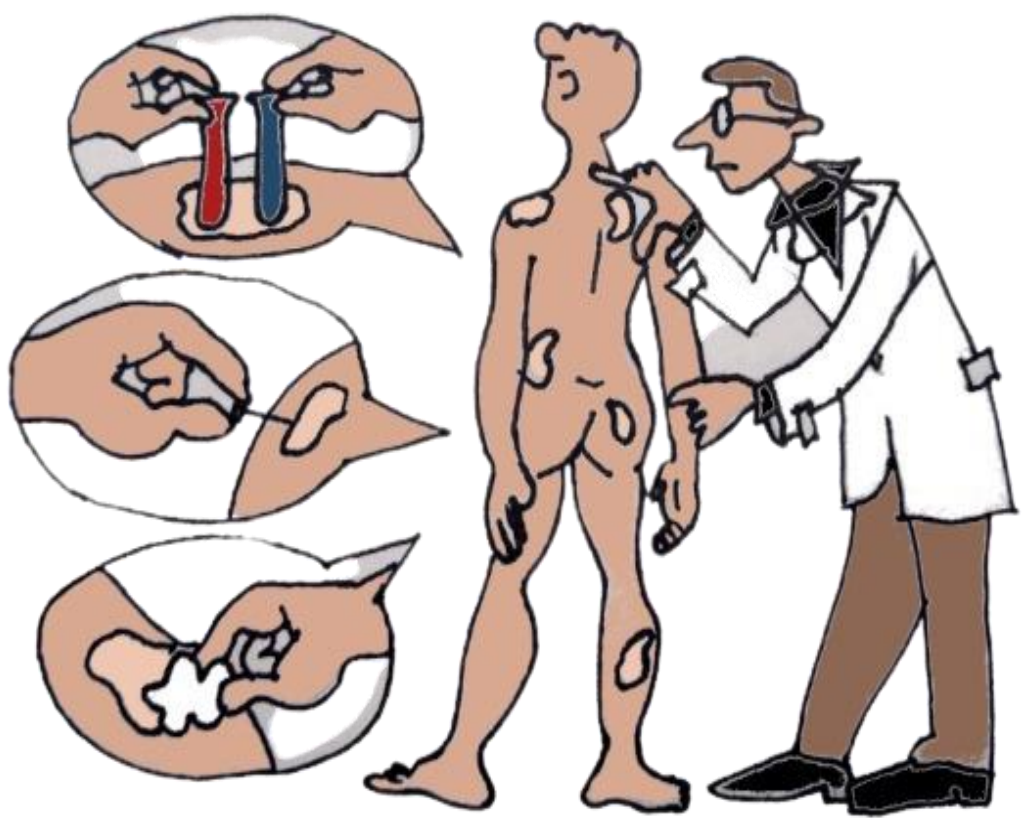




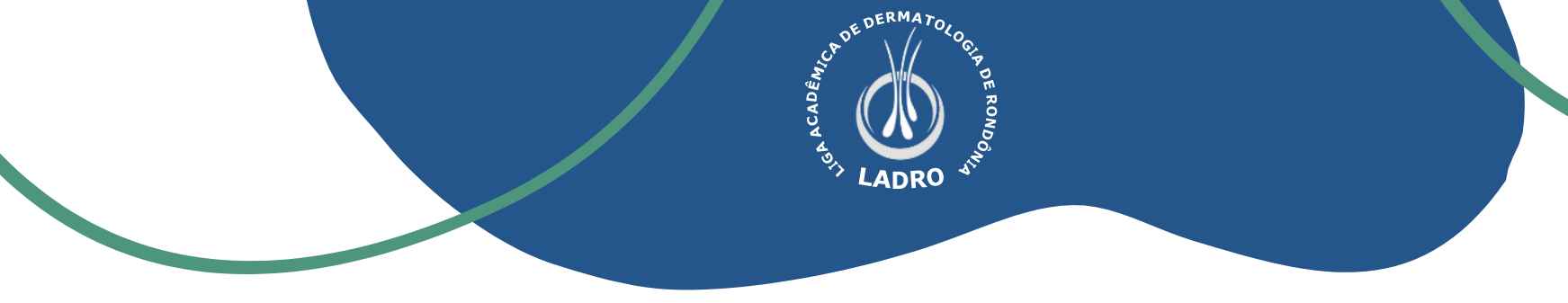

\section{COMO SE TRATA A HANSENÍASE?}

É uma doença curável, controlável e gratuito.

O tratamento será induzido de acordo com a forma da doença, ou seja,

Paucibacilar ou Multibacilar.

Lembrando que:

Paucibacilar: (Até 5 lesões e/ou 1 nervo acometido).

Multibacilar: (mais de 5 lesões e/ou 1 nervo acometido).

Tratamento Paucibacilar:

Duração: 6 a 9 meses

Esquema:

\begin{tabular}{|l|c|c|c|}
\hline & Medicamentos & $\begin{array}{c}\text { Doses Mensais } \\
\text { Supervisionadas }\end{array}$ & $\begin{array}{c}\text { Doses Diárias } \\
\text { Administradas }\end{array}$ \\
\hline Paucibacilar & Dapsona & $100 \mathrm{mg}$ & $100 \mathrm{mg}$ \\
\hline & Rifampicina & $600 \mathrm{mg}$ & ---- \\
\hline & Clofazimina & $300 \mathrm{mg}$ & 5 omg \\
\hline
\end{tabular}

Tratamento Multibacilar:

Duração: 12 a 18 meses

Esquema:

\begin{tabular}{|l|c|c|c|}
\hline & Medicamentos & $\begin{array}{c}\text { Doses Mensais } \\
\text { Supervisionadas }\end{array}$ & $\begin{array}{c}\text { Doses Diárias } \\
\text { Administradas }\end{array}$ \\
\hline Multibacilar & Dapsona & $100 \mathrm{mg}$ & $100 \mathrm{mg}$ \\
\hline & Rifampicina & $600 \mathrm{mg}$ & ---- \\
\hline & Clofazimina & $300 \mathrm{mg}$ & 5 omg \\
\hline
\end{tabular}

\section{OBSERVAÇÃO:}

Rifampicina só é administrada na dose mensal supervisionada. 


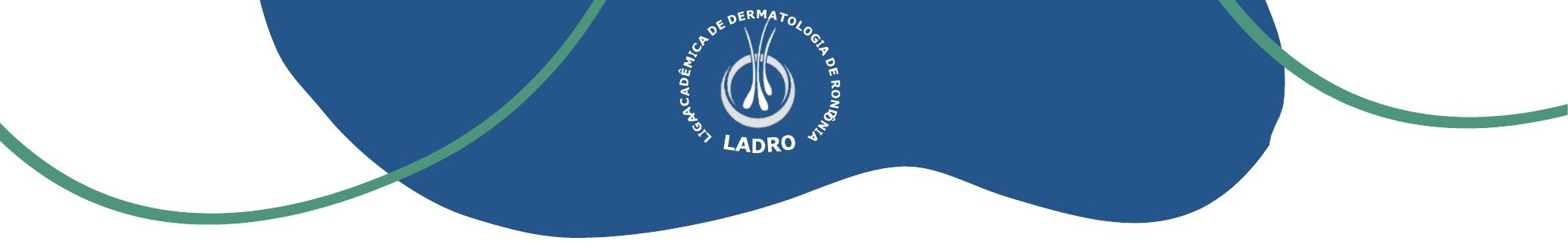

\section{CONHECENDO A MEDICAÇÃO!}

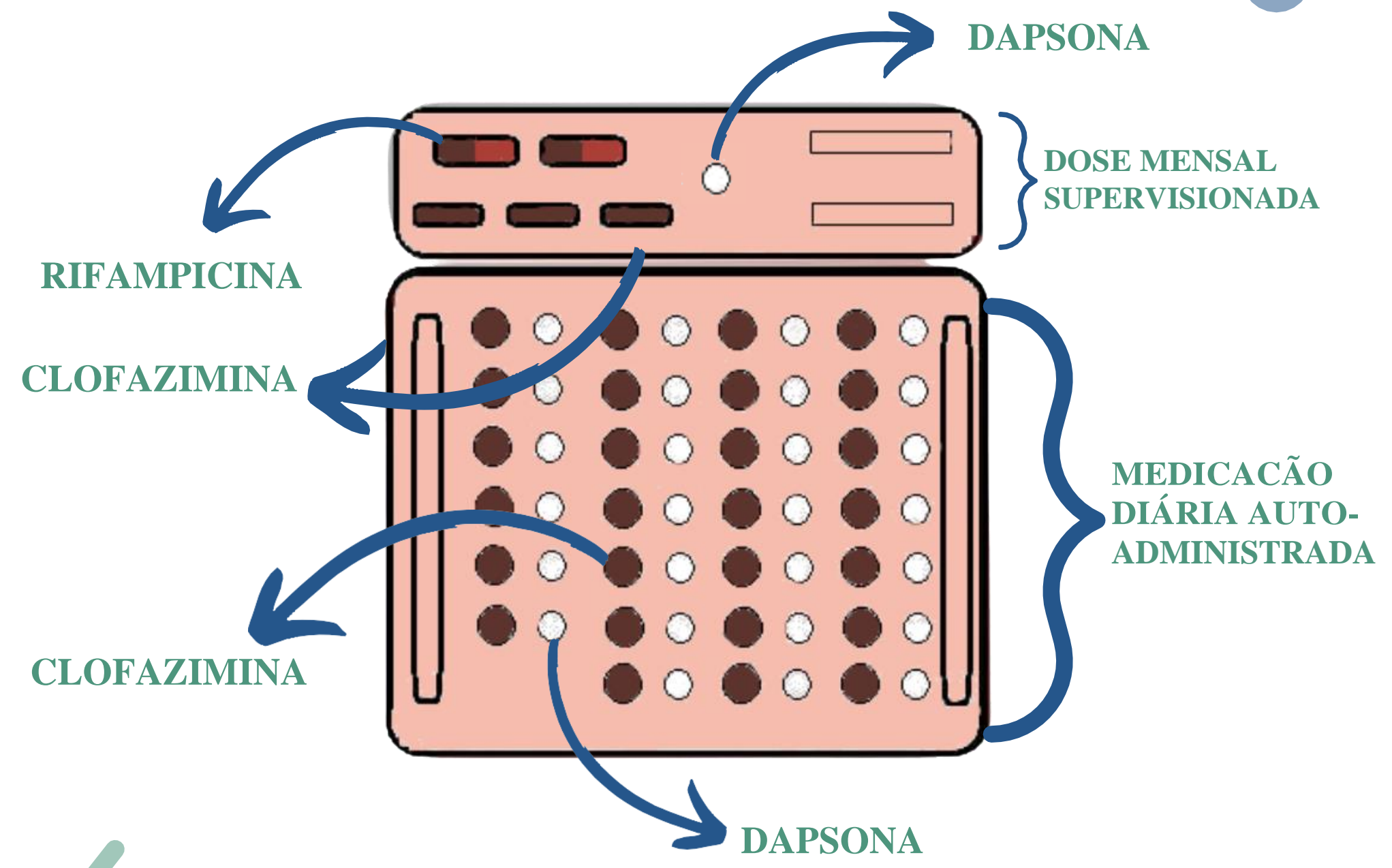




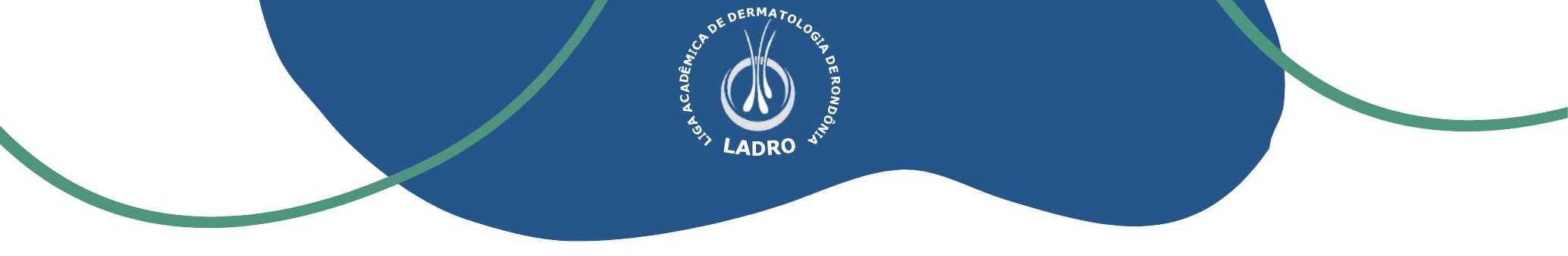

\section{CONHECENDO A MEDICAÇÃO!}

A Rifampicina é o comprimido vermelhinho tomado apenas na dose mensal supervisionada. Por isso, não estranhe se seu xixi ficar vermelho por algumas horas depois de tomar o remédio. Não sepreocupe! Não é sangue, é o remédio mesmo!

ATENÇÃO: esse medicamento pode diminuir a ação do anticoncepcional. Por isso, é importante que você e sua parceria conversem com seu médico sobre esta questão e usem preservativos nas relações!

A Clofazimina é a cápsula redondinha, marrom, que você deve tomar após o jantar. Com o passar dos meses a pele pode ficar com aspecto bronzeado e mais ressecada, principalmente se você tomar sol. Mas, não se preocupe! Depois do tratamento a cor original da sua pele vai voltando aos poucos.

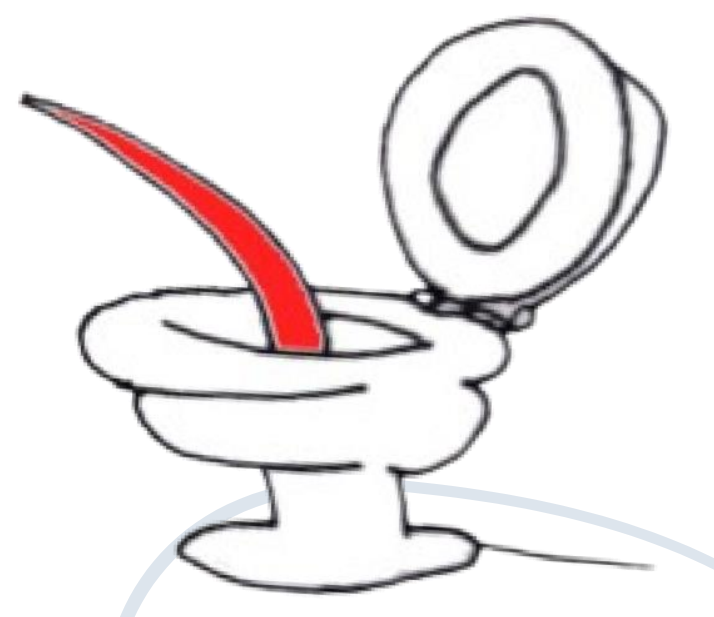




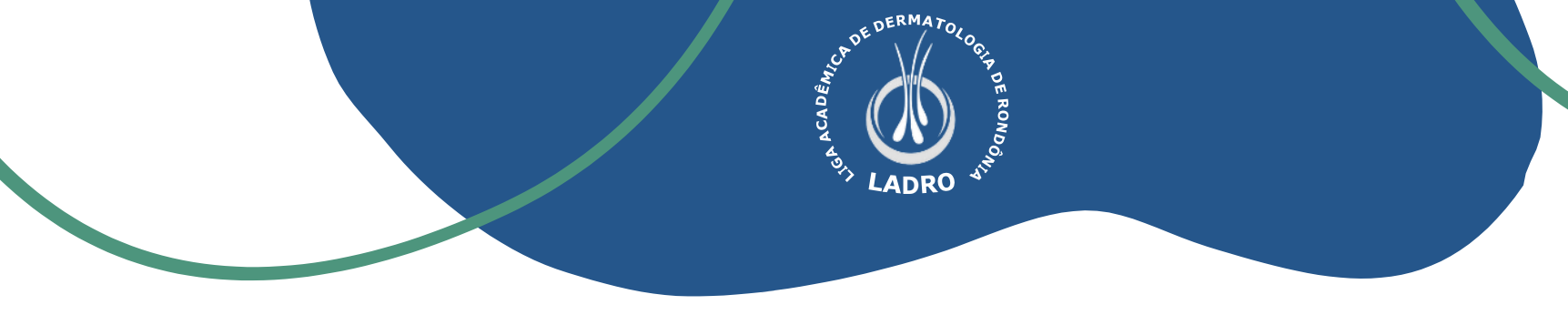

\title{
CONHECENDO A MEDICAÇÃO!
}

\begin{abstract}
A Dapsona é ocomprimido branquinho que você toma todos os dias depois do almoço. Em algumas pessoas pode causar anemia e queimação no estômago. Fique atento quanto à cor das suas mãos e dos lábios. Se você ficar mais pálido, sentir fraqueza e/ou cansaço a qualquer esforço, é hora de conversar com seu médico.
\end{abstract}

Todos esses medicamentos são antibióticos e devem ser tomados sob orientações médica.

O tratamento da hanseníase não pode ser interrompido. Sua interrupção pode provocar recaídas, reaparecimento da bactéria, e piora da doença. O medicamento não deve ser interrompido durante a gravidez e a amamentação. 


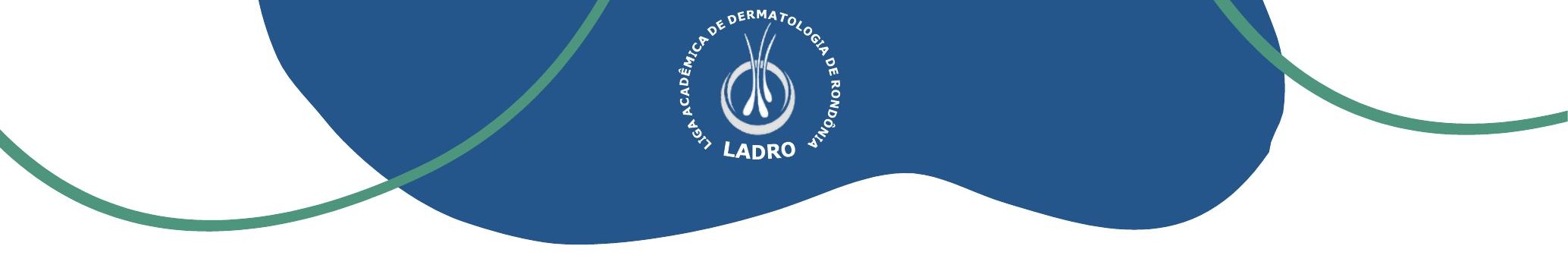

\section{O QUE SÃO REAÇÕES HANSÊNICAS?}

As reações hansênicas são processos inflamatórios agudos ou subagudos no decorrer da infecção crônica hansênica, e podem ser classificadas em dois tipos:

\section{Reação tipo 1:}

Na pele, causa novas lesões (manchas, pápulas e placas), torna lesões antigas mais inchadas e vermelhas.

Nos nervos, causa dor espontânea, devido a inflamação, resultando em dor, fraqueza e dormências (neurite).

\begin{tabular}{|c|}
\hline TIPO 1- RR \\
REAÇÃO REVERSA \\
\hline Mais frequente em Paucibacilares \\
\hline Antes, durante e mesmo no início do tratamento \\
\hline Neurites \\
\hline Hipersensibilidade \\
Tratamento: \\
Corticoides (Prednisona) \\
Descompressão dos nervos
\end{tabular}




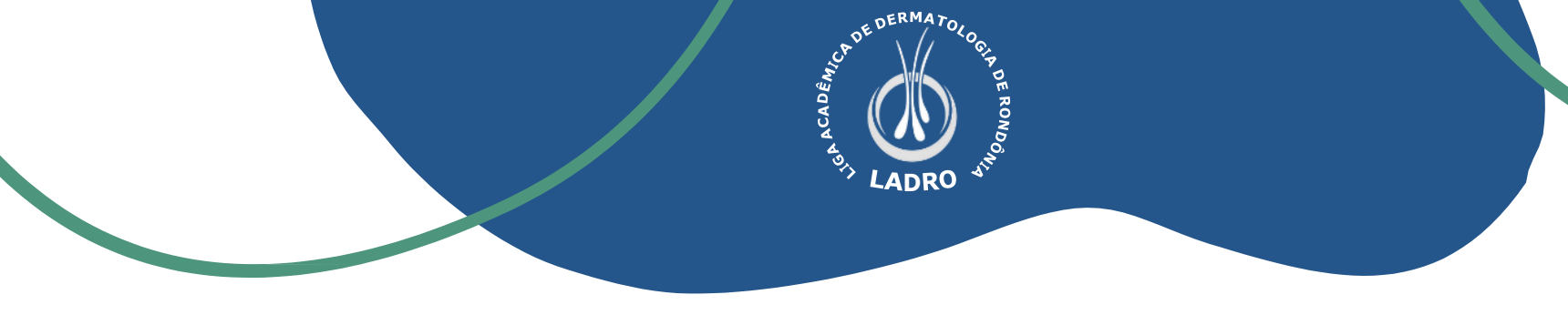

\section{Reação tipo 2:}

Na pele, causa nódulos subcutâneos dolorosos (Eritema Nodoso Hansênico- ENH). Geralmente causam manifestações sistêmicas, como: febre alta, dor articular, malestar, perda de apetite e inchaço dos pés e mãos. Pode, também, ocorrer a inflamação dos nervos.

\begin{tabular}{|c|}
\hline TIPO 2- ENH \\
ERITEMA NODOSO HANSÊNICO \\
\hline Mais frequente em Multibacilares \\
\hline Lesões de pele + neurite \\
\hline Febre e mal-estar \\
\hline Pode ser a $\mathbf{1}^{\circ}$ manifestação da doença, antes, durante ou pós \\
tratamento \\
\hline Tratamento: \\
Talidomida (exceto em mulheres em idade fértil) \\
Prednisona
\end{tabular}




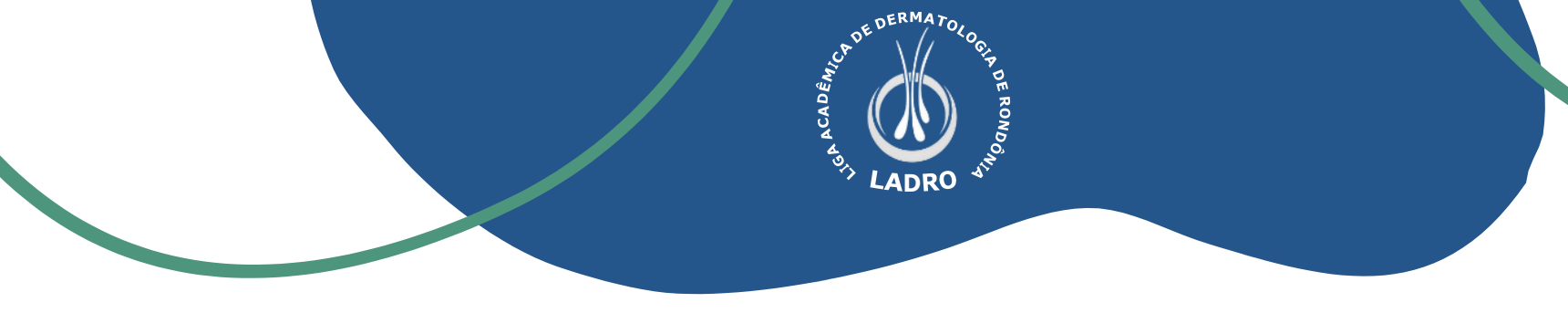

\section{CUIDADO COM AS REAÇÕES HANSÊNICAS?}

Para reações do tipo 1, o tratamento é realizado com anti-inflamatórios não hormonais e analgésico. No caso do surgimento de neurite, a Prednisona é administrado.

Pra reações do tipo 2, o tratamento é realizado com a administração de Talidomida (quando possível), anti-inflamatórios e Prednisona. É importante ressaltar que, em casos de pacientes do sexo feminino em idade fértil, a Talidomida deve ser evitada. 


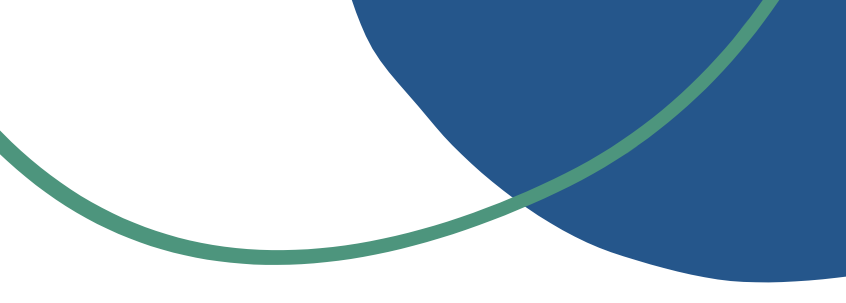

\section{COMO PREVENIR E TRATAR AS INCAPACIDADES E AS SEQUELAS FÍSICAS?}

A prevenção de incapacidades físicas baseia-se no autocuidado e é um conjunto de medidas com a tentativa de evitar a ocorrência de danos físicos, emocionais, espirituais e socioeconômicos.

Para tal, recomenda-se seguiras seguintes recomendações:

\section{Ressecamento ou dificuldade para fechar os olhos}

Usar soro fisiológico para umedecer os olhos e fazer exercícios de abrir e fechar as pálpebras com força (piscar).

Utilizar pano limpo para enxugar lágrimas, sem esfregar.

Inspeção e limpeza dos olhos toda noite com água limpa e pano limpo. Uso de colírios lubrificantes.
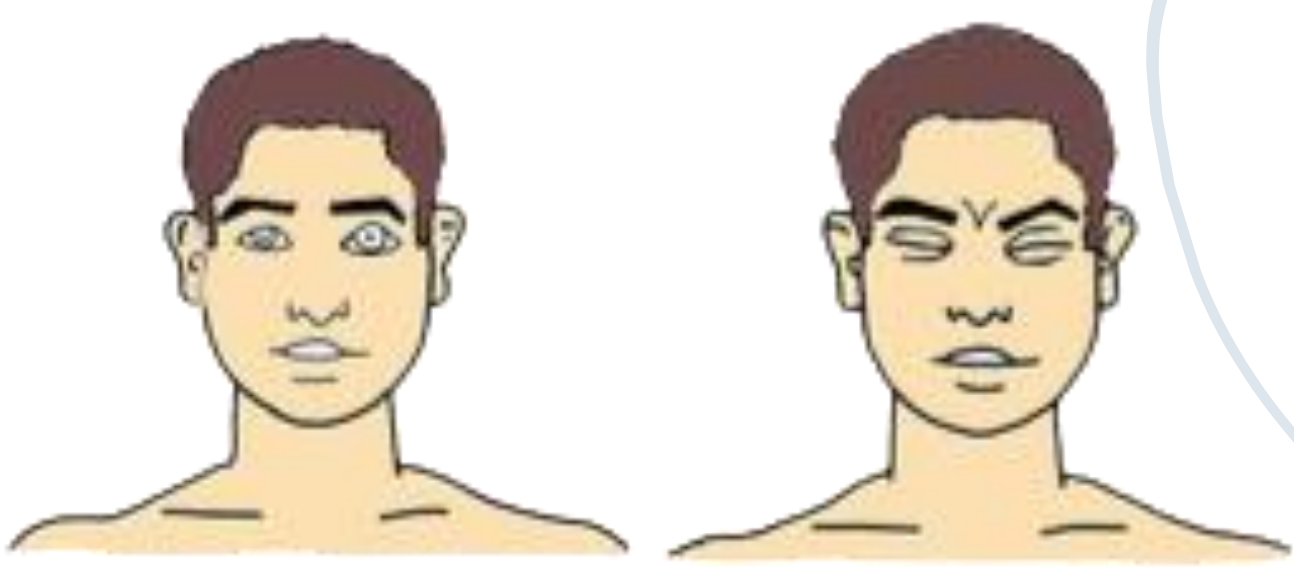


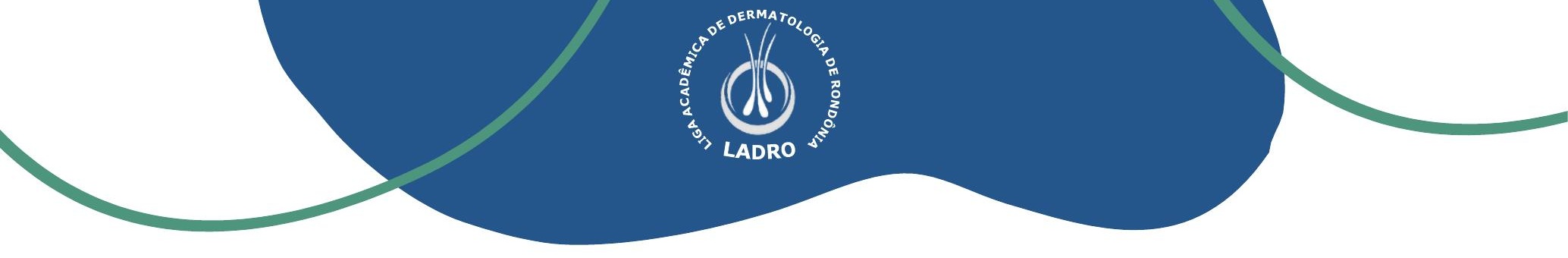

\section{Dificuldade na movimentação das mãos e dedos}

Esticar as mãos, levantando os dedos e fazendo movimentos de abre e fecha.

É indispensável o alongamento diário de mãos e pés para precaver contraturas.
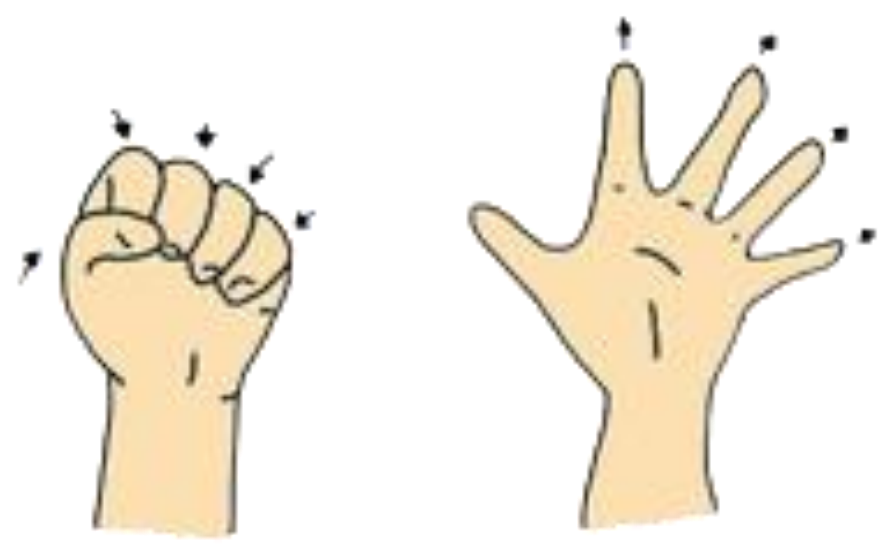

Usar luvas e meias para obter isolamento térmico, evitando bolhas e úlceras nas mãos e pés. Evitar sandálias que prendam o pé, ou sapatos apertados. O SUS tem um serviço especializado que pode fazer um calçado especialmente adequado para os seus pés. 


\begin{tabular}{|c|}
\hline Lesóes no nariz \\
\hline $\begin{array}{l}\text { Não cutucar o nariz, limpar frequentemente } \\
\text { com soro fisiológico, colocar um pouco de } \\
\text { água na palma da mão e inspirar e expirar o } \\
\text { líquido. }\end{array}$ \\
\hline
\end{tabular}

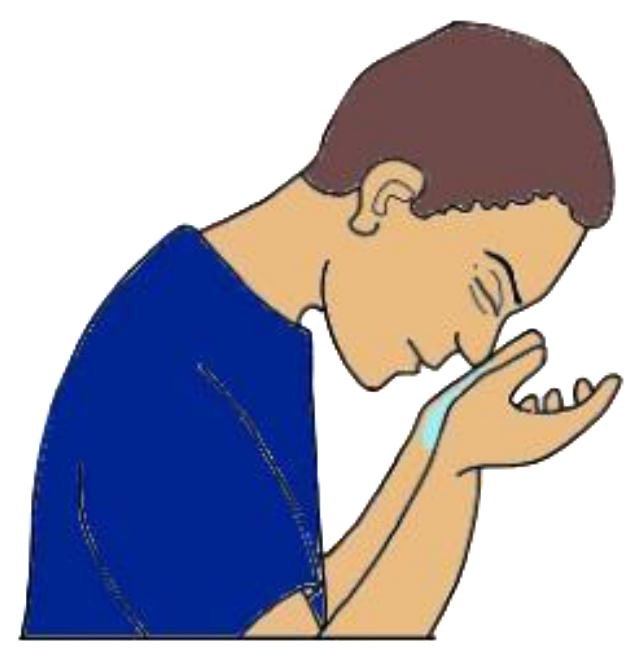

\section{Ressecamento da pele}

Hidratação e lubrificação da pele com óleos ou hidratantes.

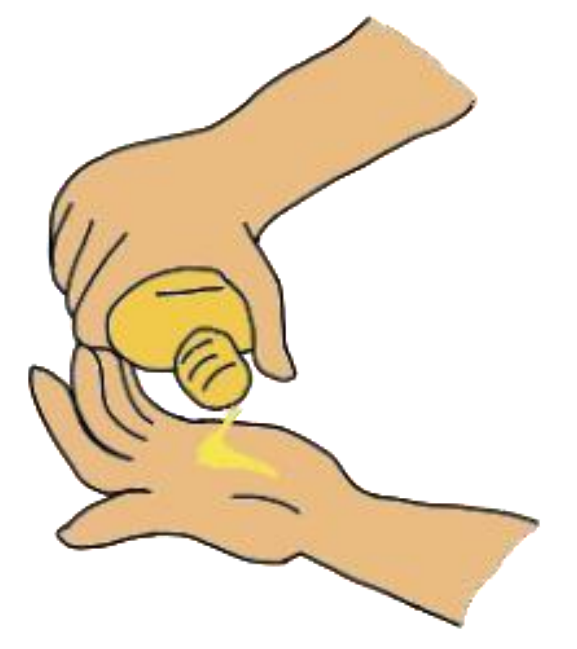

Usar chapéu com aba larga e óculos escuros com proteção contra radiação ultravioleta para não ressecar a pele. 

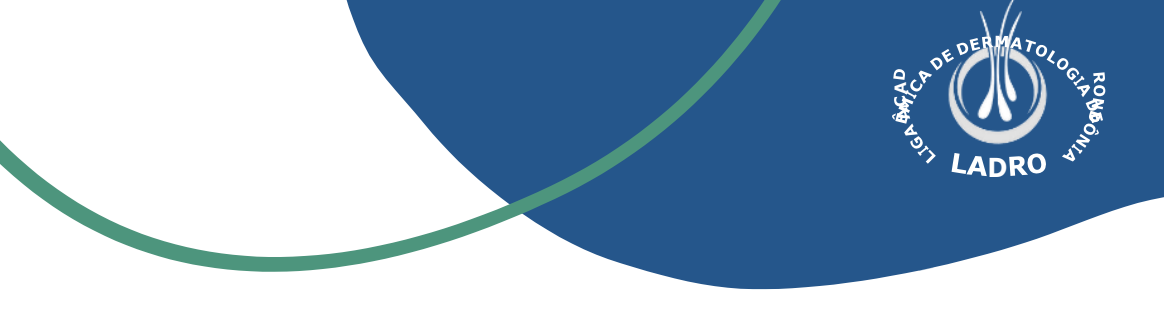

\section{A IMPORTÂNCIA DO AGENTE COMUNITÁRIO DE SAÚDE NO CONTROLE DA HANSENÍASE?}

O Agente Comunitário de Saúde (ACS) é o profissional responsável por realizar visitas domiciliares, ouvir casos comunitários, localizar problemas e agravos à saúde e notificar a equipe do plano estratégico de saúde da família sobre as necessidades das pessoas. Portanto, eles podem levar à detecção precoce da doença.

O ACS trabalha com uma equipe multidisciplinar para monitorar pacientes em tratamento de hanseníase para reduzir recorrências, identificar contatos e novos casos.

Dessa maneira, por meio de visitas domiciliares, o agente orienta o paciente com hanseníase quanto ao autocuidado, a medicação correta e a importância das visitas regulares à UBS para avaliação do processo de tratamento.

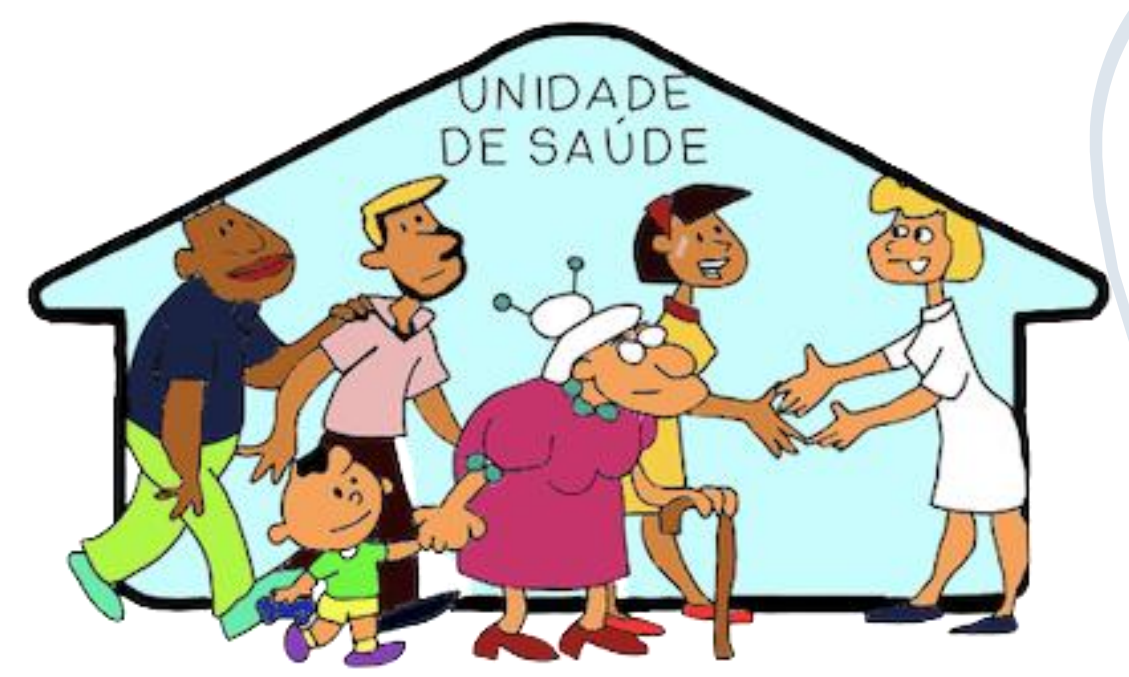




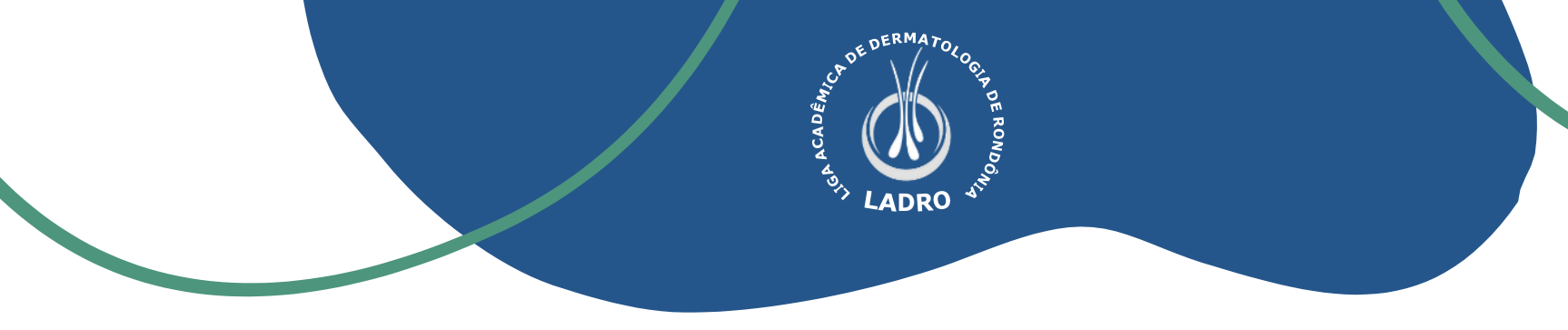

\section{COMO PROCEDER COM OS CONTACTANTES DOS PACIENTES COM HANSENÍASE?}

É considerado contato dos pacientes com hanseníase a pessoa que teve contato intradomiciliar, ou seja aquele, ou aqueles, que residem ou residiam com a pessoa contaminada com hanseníase nos últimos cinco anos.

A investigação epidemiológica das pessoas que tiveram contato intradomiciliar consiste no exame dermatoneurológico, dessa forma faz-se necessário orientar os indivíduos quanto ao período de incubação, transmissão, sinais e sintomas que podem ocorrer de forma precoce.

Deve-se ter uma atenção especial quando a investigação é feita em contatos com menores de 15 anos, pois esses foram expostos ao contágio ainda muito jovens. 


\section{CONTACTANTES}

\section{BCG}

\begin{tabular}{|c|c|}
\hline Cicatriz Vacional & Conduta \\
\hline Sem cicatriz de BCG & Fazer 1 dose \\
\hline Com 1 cicatriz de BCG & Fazer 1 dose \\
\hline Com 2 cicatrizes de BCG & Não fazer nenhuma dose a mais \\
\hline
\end{tabular}




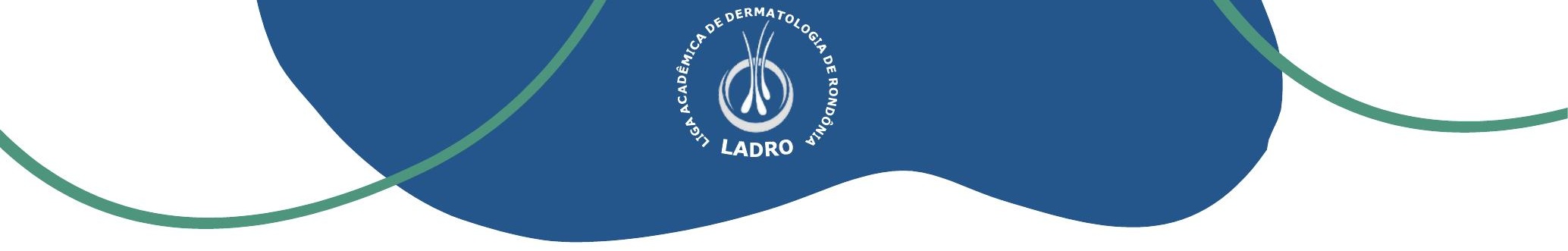

\section{VOCÊ PODE LEVAR UMA VIDA NORMAL!}

Após ter realizado o tratamento com antibióticos e estar sobre uma rotina de acompanhamento, os pacientes diagnosticados com hanseníase, estão aptos a ter uma vida normal. Ou seja, esses poderão voltar ao convívio cotidiano com seus familiares e amigos, além de estarem aptos para o retorno a suas atividades rotineiras, como estudo, esporte e trabalho

Dessa forma, podemos concluir que, apesar de algumas sequelas que podem ser deixadas devido ao acometimento da doença, o paciente, depois de realizar o seu tratamento, se encontra totalmente capaz de voltar a ter sua vida normal.

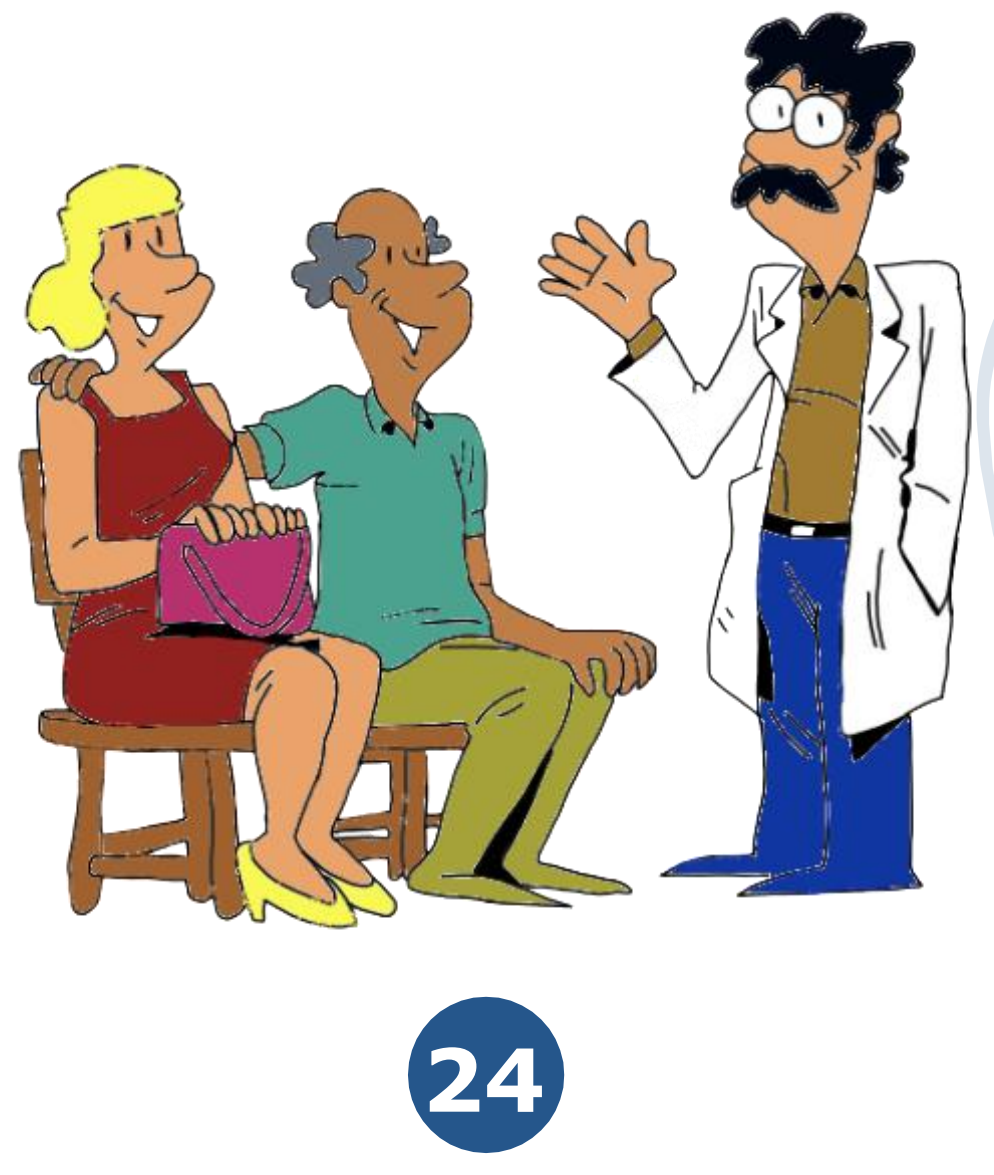




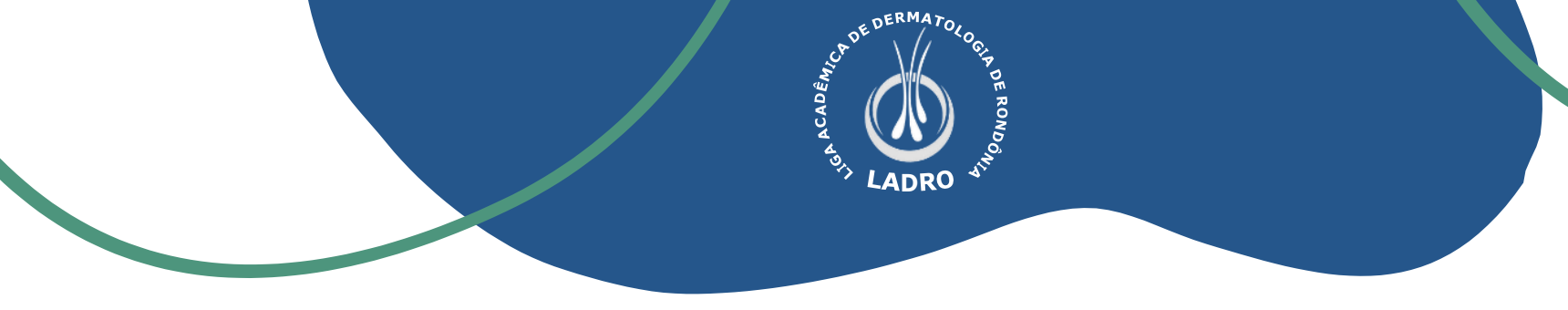

\section{REFERÊNCIAS BIBLIOGRÁFICAS}

BRASIL, Ministério da Saúde, Secretaria de Vigilância em Saúde, Departamento de Vigilância das Doenças Transmissíveis. Guia prático sobre a hanseníase. Brasília: Ministério da Saúde, 2017.

Brasil. Ministério da Saúde. Secretariade Políticas de Saúde. Departamento de Atenção Básica. Hanseníase: Atividades de controle e manual de procedimentos/ área técnica de dermatologia. Brasília (DF): Ministério da Saúde; 2001.

BRASIL, Ministérioda Saúde, Secretariade Vigilância em Saúde, Departamento de Atenção Básica - Vigilância em Saúde: Dengue, Esquistossomose, Hanseníase, Malária, Tracoma e Tuberculose- Brasília: Ministério da Saúde, 2017.

Brasil. Ministério da Saúde. Secretaria de Atenção à Saúde. Departamento de Atenção básica. Informe da Atenção Básica No 42. A responsabilidade da Atenção Básica no diagnóstico precoce da hanseníase. Brasília (DF): Ministério da Saúde; 2007.

Brasil. Ministério da Saúde. Secretaria de Atenção à Saúde. Departamento de Atenção Básica. Caderno de Atenção Básica no 21. Vigilância em Saúde: Dengue, Esquistos somose, Hanseníase, Malária, Tracoma e Tuberculose. 2aed.rev. Brasília (DF): Ministério da Saúde; 2008.

BRASIL, Ministério de Sáude. Secretária de Vigilância em Saúde. Departamento de Vigilância Epidemiológica. Autocuidado em Hanseníase: face, mãos e pés. Brasília: Editora do Ministério de Saúde, 2010.

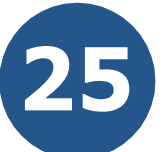


CANRIO, Djulian Diego Ribeiro do Carmo; COSTAE SILVA, Susanne Pinheiro; COSTA, Flavia Monteiro da. Saberes e práticas de agentes comunitários de saúde acerca da hanseníase. Journal of Nursing UFPE/Revista de Enfermagem UFPE,v. 8,n.1, 2014.

Coordenação Geral de Hanseníase e Doenças em Eliminação. Boletim Epidemiológico: Hanseníase.Vol.49. Secretariade Vigilância em Saúde - Brasília: Ministério da Saúde, 2018.

DE ALMEIDA GALAN, Noêmi Garcia etal. Avaliação daprática do autocuidado domiciliar em hanseníase. Hansenologia Internationalis: hanseníase e outras doenças infecciosas, v. 41, n. 1/2, p. 37-45, 2016.

DE LIMA, Jaciara Raquel Barbosa; NUNES, Daniely Oliveira; DIAS, Artur Gomes. DISTRIBUIÇÃO DA HANSENÍASE NO ESTADO DA BAHIA. Revista Científica da FASETE, p. 171, 2016.

DE GOUVÊA, Aline Russomano et al., Interrupção e abandono no tratamento da hanseníase. Brazilian Journal of Health Review, v.3,n. 4,p.10591-10603, 2020.

DIAS, Julliany Lopes et al. Características determinantes entre portadores de hanseníase em uma área hiperendêmica. Revista de Atenção à Saúde (ISSN 23594330), v. 11, n. 38, p. 32-37, 2013.

HANSEN'S Disease. Centers for Disease Control and Prevention, [S. 1.], p. https://www.cdc.gov/leprosy/index.html, 9 fev. 2017.

JOPLING, W.H. et al., Manual de hanseníase. 4. ed.São Paulo: Atheneu,1991. 


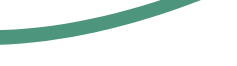

KULEVICZ, Murilo Sanches; MELLO, Tatiane de Fátima Almeida; CAPORAL, Marcelo Rodrigo. ANÁLISE EPIDEMIOLÓGICA DE CASOS DE HANSENÍASE EM UM MUNICÍPIO DO OESTE DO PARANÁ, NO PERÍODO DE 2013 A 2018. FAG JOURNAL OF HEALTH (FJH), v. 3, n.2, p. 186-189, 2021.

LEITE, Cleber Queiroz; SANTOS, Brian França dos. EDUCAÇÃO EM SAÚDE FRENTE AO PRECONCEITO DA HANSENÍASE E SEUS ESTIGMAS SOCIAIS.. In: COSTA, Ana Carolina Messias de Souza Ferreira (Org.). Internacional Saúde Única (Interface Mundial) Recife: Even3, 2021.

MARTINS, Rosa Maria Grangeiro et al., Desenvolvimento de uma cartilha para a promoção do autocuidado na hanseníase. Rev.enferm. UFPEon line, p.[1-7], 2019.

OLIVEIRA, M. L. de W. et al., Hanseníase: cuidados para evitar complicações. Brasília: Fundação Nacional de Saúde, 1998. 32 p.

OLIVEIRA, Joana; MARINUS, Maria Wanderleya de Lavor Coriolano; MONTEIRO, Estela Maria Leite Meirelles. Práticas de atenção à saúde de crianças e adolescentes com hanseníase: discursos de profissionais. Revista Gaúcha de Enfermagem, v.41, 2020.

OPROMOLLA, D.V. A. As Incapacidades na Hanseníase. In: . Noções de Hansenologia. Bauru: Centro de Estudos Dr. Reynaldo Quagliato/Hospital Lauro de Souza Lima, 1981.

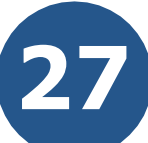


SAVASSI, Leonardo Cançado Monteiro et al., Atenção Primária e Hanseníase: desafios educacionais e assistenciais. 2013. Tese de Doutorado.

SOARES, Francisco Mayron Morais et al., Construção de tecnologias em enfermagem para à promoção da saúde portadores de hanseníase. 2016.

VIETH, Hannelore et al., Guia de prevenção ocular em hanseníse. [S.1.]:Talmilep/Dahw/Centro de Prevenção Oftalmológica/Instituto Lauro de Souza Lima,[20--?]. 54 p. 


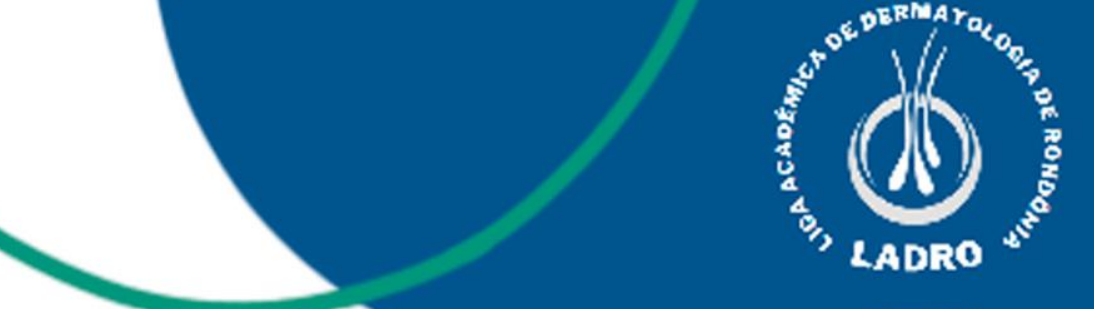

\section{GUIA PRÁTICO DA} HANSENÍASE NA ATENÇÃo BÁSICA

$1^{\circ}$ Edição

A hanseníase, endemia típica, mas não exclusiva do Brasil, tem como principal protagonista a desinformação da população e mesmo de profissionais da saúde. Este guia tem por objetivo modesto auxiliar no preenchimento desta lacuna de informação. Assim sendo, abrange de forma clara e concisa, especialmente: informações sobre o que é hanseníase, seus sinais e sintomas iniciais, como se transmite a doença, como previne suas complicações, como é realizado o diagnóstico e, consequentemente, como se trata a hanseníase. Traz, ainda, informações de como a medicação deverá ser administrada, bem como os eventuais efeitos adversos mais comuns de cada medicamento. Explica ainda, de maneira resumida, como proceder com os contatos dos pacientes com hanseníase.

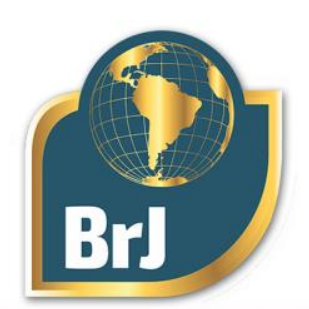

